\title{
Numerical analysis of a finite element formulation of the P2D model for Lithium-ion cells
}

\author{
R. Bermejo ${ }^{1}$
}

Received: 19 February 2020 / Revised: 31 August 2021 / Accepted: 2 October 2021 /

Published online: 23 October 2021

(c) The Author(s) 2021

\begin{abstract}
The mathematical P2D model is a system of strongly coupled nonlinear parabolicelliptic equations that describes the electrodynamics of lithium-ion batteries. In this paper, we present the numerical analysis of a finite element-implicit Euler scheme for such a model. We obtain error estimates for both the spatially semidiscrete and the fully discrete systems of equations, and establish the existence and uniqueness of the fully discrete solution.
\end{abstract}

\section{Introduction}

In this paper, we present the numerical analysis of a finite element-implicit Euler method to calculate the numerical solution of the so called pseudo-two-dimensional (P2D) model proposed by J. Newman and coworkers [3]. This is a mathematical model based on the electrochemical kinetics and continuun mechanics laws, which consists of a system of coupled nonlinear parabolic-elliptic equations to model the physicalchemical phenomena governing the behavior of lithium ion batteries. The P2D model is very much used in engineering studies. A good presentation of it can be found in [13] and [15]. A lithium-ion battery system is composed of a number of lithium-ion cells. A typical cell consists of three regions, namely, a porous negative electrode (which plays the role of anode of the cell in the discharge process) connected to the negative terminal collector of the battery, a separator that is an electron insulator allowing the flow of lithium ions between the anode and the cathode, and a porous positive electrode (which plays the role of cathode during the discharge process) connected to the positive terminal, see Fig. 1. We must point out that in the charge process the negative electrode plays the role of cathode and the positive electrode is the anode. The

$凶 \quad$ R. Bermejo

rodolfo.bermejo@upm.es

1 Dpto. Matemática Aplicada a la Ingeniería Industrial, ETSII Universidad Politécnica de Madrid, Madrid, Spain 


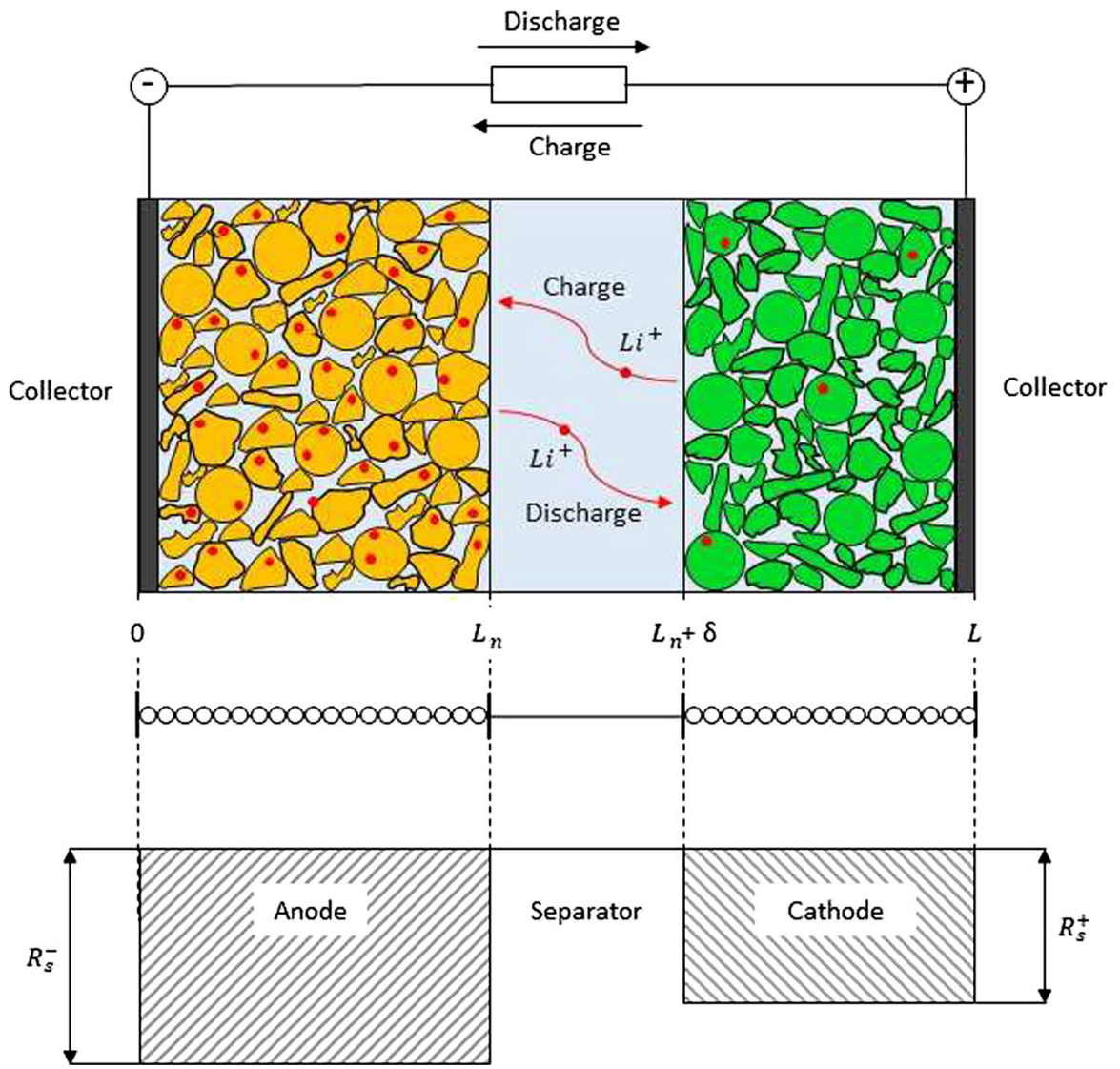

Fig. 1 Upper panel: a cross-section of a cell along the $\mathrm{x}$-direction. The lithium ions travel from the anode to the cathode during the discharge process and in the opposite direction during the charge process. Middel panel: the cell model as a non-denumerable collection of solid spheres plus the separator, there is one sphere of radius $R_{S}(x)$ at each point $x$ of the electrodes. Lower panel: the domain $\mathrm{D}_{3}$

electrodes are composite porous structures of highly packed active lithium particles, typically $\mathrm{Li}_{\mathrm{x}} \mathrm{C}_{6}$ in the negative electrode and metal oxide, such as $\mathrm{Li}_{1-\mathrm{x}} \mathrm{Mn}_{2} \mathrm{O}_{4}$, in the positive electrode, plus a binder and a polymer that act as conductive agents. Furthermore, the cell is filled with the electrolyte that occupies the holes left free by the particles and the filler material. The electrolyte is a lithium salt dissolved in an organic solvent. In the description of the model it is customary to consider two phases: the electrolyte phase and the solid phase, the latter is composed of the solid particles of the electrodes.

The P2D model of a lithium-ion cell considers that the dynamics is only relevant along the $x$-axis, neglecting what happens along the $y$-axis and $z$-axis, because the ratios $\frac{L_{x}}{L_{y}}$ and $\frac{L_{x}}{L_{z}}=O\left(10^{-3}\right), L_{x}, L_{y}$ and $L_{z}$ being the characteristic length scales along the corresponding axes. The main modeling assumptions are the following : (1) The active particles of the electrodes are assumed to be spheres of radius $R_{S}$ which 
may be different in each electrode. (2) Side reactions are neglected and no gas phase is present. (3) The transport of lithium ions is due to diffusion and migration in the electrolyte solution, and in the solid particles the atoms of lithium move between vacancies in the crystalline structure of the particles due to local diffusion in concentration. By longitudinal and latitudinal symmetry considerations, the diffusion in the active particles is only in the radial direction. (4) The electrochemical reaction of lithium insertion and extraction processes follows the Buttler-Volmer law. (5) The effective transport coefficients are calculated by the Bruggeman relation, i.e., $\mu^{\text {eff }}=\mu \varepsilon^{p}(\mathrm{p}=1.5)$, where $\mu$ is a generic transport coefficient and $\varepsilon$ is the component volume fraction of the material in the composite electrodes and separator.To formulate the equations of the model we distinguish the following domains.

$$
\left\{\begin{array}{l}
D_{\mathrm{n}}=\left(0, L_{n}\right), D_{\mathrm{s}}=\left(L_{n}, L_{n}+\delta\right), D_{\mathrm{p}}=\left(L_{n}+\delta, L\right), L_{p}:=L-\left(L_{n}+\delta\right), \\
D_{1}=(0, L), D_{2}=D_{\mathrm{n}} \cup D_{\mathrm{p}} \text { and } D_{3}=\cup_{x \in D_{2}}\{x\} \times\left(0, R_{\mathrm{S}}(x)\right),
\end{array}\right.
$$

where $D_{\mathrm{n}}, D_{\mathrm{s}}$ and $D_{\mathrm{p}}$ denote the domains of the negative electrode, the separator and the positive electrode respectively. Notice that $D_{1}$ represents the cell domain, $D_{2}$ is a domain that is the union of two disjoint domains corresponding to the electrodes, and $D_{3}$ is in a certain sense a modeling space accounting for the spherical balls of radius $R_{\mathrm{S}}(x)$ that represent at each $x \in D_{2}$ the solid active particles, such that when $x \in D_{\mathrm{n}}, R_{\mathrm{S}}(x)=R_{\mathrm{S}}^{-}$, and when $x \in D_{\mathrm{p}}, R_{\mathrm{S}}(x)=R_{\mathrm{S}}^{+}$. The variables of the model are the following: for the electrolyte phase, the molar concentration of lithium ions $u(x, t)$, and the electric potential $\phi_{1}(x, t), x \in D_{1}$; for the solid phase, the molar concentration of lithium $v(x ; r, t), x \in D_{2}$ and $r \in\left(0 ; R_{\mathrm{S}}(x)\right)$, and the electric potential $\phi_{2}(x, t), x \in D_{2}$. Another important variable is the so called molar flux of lithium ions exiting the solid particles, $J\left(x, u, v, \phi_{1}, \phi_{2}, U\right) / F, F$ being the Faraday constant. The mathematical expression of $J$ is given by the Buttler-Volmer law, see (1).

Many numerical models to integrate the P2D model have been proposed. The first one is the Dualfoil model developed by J. Newman and his collaborators [12], this is a model that uses second order finite differences for space discretization of the differential operators combined with the first order backward Euler time stepping scheme; the Dualfoil model is distributed as free software, which is being updated through time. Later on, authors such as [11] and [17], just to cite a few, have developed their own codes by using second order finite volume for space discretizations combined with the first order in time implicit Euler scheme for time discretization. Other authors make the numerical simulations with COMSOL multi-physics package that uses finite elements for space discretizations of the equations, the resulting system of nonlinear differential equations is integrated by different time stepping schemes, in particular, conventional DAE solvers, such as DASK [14]. New numerical models have recently been proposed to improve the computational efficiency, to this respect, we mention the operator splitting technique of [6], the orthogonal collocation method for space discretization combined with the first order implicit Euler scheme for time discretization of [9], and the implicit-explicit Runge-Kutta-Chebyshev finite element method of [1]. Despite the activity in the development of numerical methods no rigorous numerical 
analysis of such methods has been published so far; so, to the best of our knowledge, this is the first paper presenting the analysis of a numerical method developed to integrate the $\mathrm{P} 2 \mathrm{D}$ model.

The layout of the paper is the following. In Sect. 2 we introduce the governing equations of the P2D model together with the functional framework needed for the numerical analysis. Section 3 is devoted to the semidiscrete space discretization of the model in a finite element framework. The error analysis of the semi-discrete solution is performed in Sect. 4. Since this analysis is long, then we have split the section into three subsections in order to make more palatable its presentation. Section 4.1 is a collection of auxiliary results; Sections 4.2 and 4.3 deal with the error estimates for the potentials and the concentrations, respectively. The fully discrete model and its error analysis is presented in Sect. 5, which is also split into subsections. Since the fully discrete model is a nonlinear system of elliptic and fully discrete parabolic equations at each time instant $t_{n}$, then we have also studied the existence and uniqueness of the solution by applying Minty-Browder theorem [18] for the elliptic equations, and Brower's fixed point theorem for the parabolic equations.

\section{The governing equations of the isothermal P2D model}

We consider the governing equations of the isothermal P2D model for the variables $u(x, t), v(x ; r, t), \phi_{1}(x, t)$ and $\phi_{2}(x, t)$ presented in Chapters 3 and 4 of [15]. However, to facilitate both the formulation of the numerical method to integrate these equations and its numerical analysis, it is convenient to make the changes of variable introduced in [10] and [19]. Thus, in order to make homogeneous the Neumann type boundary conditions for the potential $\phi_{2}$ one considers the function $H(x, t)$ given by the expression

$$
H(x, t)= \begin{cases}-\frac{\left(x-L_{n}\right)^{2} I(t)}{2 \sigma L_{n} A}, & x \in D_{\mathrm{n}}, \\ \frac{\left(x-\left(L_{n}+\delta\right)\right)^{2} I(t)}{2 \sigma L_{p} A}, & x \in D_{\mathrm{p}},\end{cases}
$$

where $I(t)$ denotes the applied current, $A$ is the area of the plate and $\sigma$ is a positive coefficient defined below, and replace $\phi_{2}(x, t)$ by $\phi_{2}(x, t)+H(x, t)$; likewise, we replace the potential $\phi_{1}(x, t)$ by $\phi_{1}(x, t)+\alpha \ln u(x, t)$, with $\alpha=\alpha(u)=\frac{2 R T \kappa(u)}{F}\left(t_{+}^{0}-1\right)$, where $\kappa(u)>0$ denotes the effective electrolyte phase ionic conductivity; $t_{+}^{0}>0$ is the so called transfer number, which is assumed to be constant; $R$ is the universal gas constant and $T$ denotes the absolute temperature inside the cell, which is assumed to be constant in the isothermal model; this latter change of variable for $\phi_{1}(x, t)$ simplifies the expression of the equation for the potential of the electrolyte phase written in Chapter 4 of [15], making it more manageable from a computational viewpoint. Another important variable, as we mentioned above, is the reaction current density $J$. The 
reaction rate is coupled to phase potentials by the Buttler-Volmer kinetic expression.

$$
J=J\left(x, u, v_{s}, T, \eta\right)=\left\{\begin{array}{l}
a_{s} i_{0}\left(\exp \frac{\alpha_{a} F}{R T}\left(\eta-\frac{R_{S E I}}{a_{s}} J\right)\right. \\
\left.-\exp \frac{-\alpha_{c} F}{R T}\left(\eta-\frac{R_{S E I}}{a_{s}} J\right)\right) \text { if } x \in D_{n} \cup D_{p}, \\
0 \text { if } x \in D_{s} .
\end{array}\right.
$$

In this expression, $v_{s}=v\left(x ; R_{S}(x), t\right)$ denotes the lithium concentration on the surface of the active particles; $a_{s}=a_{s}(x)=\frac{3 \varepsilon_{s}(x)}{R_{S}(x)}$ is the active area per electrode unit volume; $\varepsilon_{s}(x)$ denotes the volume fraction of the active material, $\varepsilon_{s}(x)=\varepsilon_{s}^{-}>0$ for $x \in D_{n}$ and $\varepsilon_{s}(x)=\varepsilon_{s}^{+}>0$ for $x \in D_{p} ; \alpha_{a} \in(0,1)$ and $\alpha_{c} \in(0,1)$ are anodic and cathodic transfer coefficients for an electron reaction; $R_{S E I}$ represents the solid interface resistance, usually, $R_{S E I}=0$ in the engineering literature unless the model also considers aging phenomena of the battery, so in this paper we take $R_{S E I}=0$.

$$
\eta=\eta\left(x, \phi_{1}, \phi_{2}, U\right)=\left\{\begin{array}{l}
\phi_{2}(x, t)-\phi_{1}(x, t)-U\left(x, v_{s}\right) \text { if } x \in D_{n} \cup D_{p}, \\
0 \text { if } x \in D_{s},
\end{array}\right.
$$

where $U$ stands for the equilibrium potential at the solid electrolyte interface, which is assumed to be known. $i_{0}$ is the exchange current density, i.e.,

$$
i_{0}=i_{0}\left(u, v_{s}\right)=k u^{\alpha_{a}}\left(v_{\max }-v_{s}\right)^{\alpha_{a}} v_{s}^{\alpha_{c}} \text { if } x \in D_{n} \cup L_{n},
$$

here, $v_{\max }$ is the maximum concentration of lithium in the solid phase, which may have different values in the positive and negative electrodes, so

$$
v_{\max }=v_{\max }(x)=\left\{\begin{array}{l}
v_{\max }^{-} \text {if } x \in D_{n}, \\
v_{\max }^{+} \text {if } x \in D_{p}
\end{array}\right.
$$

the coefficient $k$ represents the kinetic rate constant,

$$
k=k(x)=\left\{\begin{array}{l}
k^{-} \text {if } x \in D_{n} \\
k^{+} \text {if } x \in D_{p}
\end{array}\right.
$$

Considering the above mentioned changes of variable and taking the transfer coefficients $\alpha_{a}$ and $\alpha_{c}$ equal to 0.5 , as many engineering papers do, the expression for the reaction current that we use in the paper is

$$
J=J\left(x, u, v_{s}, \phi_{1}, \phi_{2}, \bar{U}\right)=\left\{\begin{array}{l}
a_{2}(x) i_{0} \sinh (\beta \eta), \forall x \in D_{2}, \\
0 \text { for } x \notin D_{2},
\end{array}\right.
$$


where $\beta=\frac{F}{2 R T} ; a_{2}(x)=3 \varepsilon_{S}(x) / R_{S}(x)$; and

$$
\eta=\phi_{2}-\phi_{1}-\alpha \ln u-\bar{U}
$$

$\bar{U}=\bar{U}\left(x, t, v_{s}\right)=U\left(v_{s}\right)-H(x, t)$. Noting that the boundaries $\partial D_{1}$ and $\partial D_{2}$ of the domains $D_{1}$ and $D_{2}$ are $\partial D_{1}:=\{0, L\}$ and $\partial D_{2}:=\left\{0, L_{n}, L_{n}+\delta, L\right\}$, we formulate the equations of the model as follows.

Concentration $u(x, t)$ in the electrolyte phase.

$$
\left\{\begin{array}{l}
\frac{\partial u}{\partial t}-\frac{\partial}{\partial x}\left(k_{1} \frac{\partial u}{\partial x}\right)=a_{1}(x) J \text { in } D_{1} \times\left(0, T_{\mathrm{end}}\right) \\
\left.\frac{\partial u}{\partial x}\right|_{\partial D_{1} \times\left(0, T_{\mathrm{end}}\right)}=0, u(x, 0)=u^{0}(x) \text { in } D_{1}
\end{array}\right.
$$

Concentration $v(x ; r, t)$ in the solid phase. For almost every $x \in D_{2}$,

$$
\left\{\begin{array}{l}
\frac{\partial v}{\partial t}-\frac{k_{2}}{r^{2}} \frac{\partial}{\partial r}\left(r^{2} \frac{\partial v}{\partial r}\right) v=0 \text { in } D_{3} \times\left(0, T_{\mathrm{end}}\right), \\
\left.\frac{\partial v}{\partial r}\right|_{r=0}=0,\left.k_{2} \frac{\partial v}{\partial r}\right|_{r=R_{\mathrm{S}}(x)}=\frac{-J}{a_{2}(x) F}, v(x ; r, 0)=v^{0}(x ; r) \text { in } D_{3} .
\end{array}\right.
$$

Electrolyte potential $\phi_{1}(x, t)$.

$$
\left\{\begin{array}{l}
-\frac{\partial}{\partial x}\left(\kappa(u) \frac{\partial \phi_{1}}{\partial x}\right)=J \text { in } D_{1} \times\left(0, T_{\text {end }}\right) \\
\left.\frac{\partial \phi_{1}}{\partial x}\right|_{\partial D_{1} \times\left(0, T_{\text {end }}\right)}=0 \\
\int_{D_{1}} \phi_{1}(x, t) d x=0
\end{array}\right.
$$


Solid phase potential $\phi_{2}(x, t)$.

$$
\left\{\begin{array}{l}
\frac{\partial}{\partial x}\left(\sigma \frac{\partial \phi_{2}}{\partial x}\right)=J+g \text { in } D_{2} \times\left(0, T_{\mathrm{end}}\right), \\
\left.\sigma \frac{\partial \phi_{2}}{\partial x}\right|_{\partial D_{2} \times\left(0, T_{\mathrm{end}}\right)}=0, \\
g(x, t)=\left\{\begin{array}{c}
\frac{-I(t)}{L_{n} A}, x \in D_{\mathrm{n}}, \\
\frac{I(t)}{L_{p} A}, x \in D_{\mathrm{p}},
\end{array}\right.
\end{array}\right.
$$

where $a_{1}(x)=\frac{1-t_{+}^{0}}{3 \varepsilon_{s}(x) F}$. In these equations, $k_{1}(x)>0$ and $k_{2}(x)>0$ represent effective diffusion coefficients in the electrolyte and solid phases respectively, and $\sigma(x)$ denotes the effective electric conductivity in the solid phase. The functions $a_{1}(x), a_{2}(x), \sigma(x)$ and $k_{2}(x)$ are considered to be piecewise positive constant functions in the sense that they have different constant values in the negative electrode, separator and positive electrode.

We also have to consider that for $t \in\left(0, T_{\text {end }}\right), J\left(x, u, v_{\mathrm{s}}, \phi_{1}, \phi_{2}\right)$ satisfies the algebraic conditions

$$
\left\{\begin{array}{l}
\int_{D_{1}} J d x=\int_{D_{2}} J d x=0 \\
\int_{D_{\mathrm{n}}} J d x=I(t), \int_{D_{\mathrm{p}}} J d x=-I(t) .
\end{array}\right.
$$

Notice that the first row of algebraic conditions follow directly from (7) and the definition of $J$, whereas the second row conditions translates the boundary conditions of the solid phase potential. It is worth remarking the conservative properties enjoyed by both $u(x, t)$ and $v(x ; r, t)$; namely, for all $t \in\left[0, T_{\text {end }}\right]$

$$
\int_{D_{1}} u(x, t) d x=\int_{D_{1}} u(x, 0) d x
$$

and

$$
\int_{D_{2}} \int_{0}^{R_{S}(x)} v(x ; r, t) r^{2} d r d x=\int_{D_{2}} \int_{0}^{R_{S}(x)} v(x ; r, 0) r^{2} d r d x
$$


These relations are readily obtained by integrating (5) and (6) and using the corresponding boundary conditions. Moreover, it can be shown [10] that for $t \geq 0$ and $x \in D_{1}, u(x, t)>0$, similarly, for $(x, r) \in D_{3}, 0<v(x ; r, t)<v_{\max }$.

Let $D$ denote a generic open bounded domain in $\mathbb{R}$; hereafter, the closure of a domain $D$ is denoted $\bar{D}$. The functional spaces that we use in this paper are the following. The Sobolev spaces $H^{m}(D), m$ being a nonnegative integer, when $m=0, H^{0}(D):=$ $L^{2}(D)$; the Lebesgue spaces $L^{p}(D), 1 \leq p \leq \infty$; the spaces of measurable radial functions [4]

$$
H_{r}^{q}(0, R):=\left\{v:(0, R) \rightarrow \mathbb{R}:\|v\|_{H_{r}^{q}(0, R)}^{2}=\sum_{j=0}^{q} \int_{0}^{R}\left(\frac{d^{j} v}{d r^{j}}\right)^{2} r^{2} d r<\infty\right\}
$$

$q$ being a nonnegative integer, when $q=0$ we set $H_{r}^{0}(0, R):=L_{r}^{2}(0, R)$; also, for $p$ being a nonnegative integer, the normed spaces of measurable functions

$$
H^{p}\left(D_{2} ; H_{r}^{q}\left(0, R_{s}(\cdot)\right)\right):=\left\{v: D_{2} \rightarrow H_{r}^{q}\left(0, R_{s}(\cdot)\right):\|v\|_{H^{p}\left(D_{2} ; H_{r}^{q}\left(0, R_{s}(\cdot)\right)\right)}<\infty\right\}
$$

where $\|v\|_{H^{p}\left(D_{2} ; H_{r}^{q}\left(0, R_{s}(\cdot)\right)\right)}^{2}=\sum_{j=0}^{p} \int_{D_{2}}\left\|\frac{\partial^{j} v(x ; \cdot)}{\partial x^{j}}\right\|_{H_{r}^{q}\left(0, R_{s}(x)\right)}^{2} d x$; and the spaces

$$
H_{r}^{p, q}\left(D_{2} \times\left(0, R_{S}(\cdot)\right)\right)=H^{p}\left(D_{2}, L_{r}^{2}\left(0, R_{S}(\cdot)\right)\right) \cap L^{2}\left(D_{2}, H_{r}^{q}\left(0, R_{S}(\cdot)\right)\right)
$$

with norm

$$
\|u\|_{H_{r}^{p, q}\left(D_{2} \times\left(0, R_{s}(\cdot)\right)\right)}^{2}:=\|u\|_{H^{p}\left(D_{2}, L_{r}^{2}\left(0, R_{S}(\cdot)\right)\right)}^{2}+\|u\|_{L^{2}\left(D_{2}, H_{r}^{q}\left(0, R_{s}(\cdot)\right)\right)}^{2} .
$$

Notice that $H_{r}^{0,0}\left(D_{2} \times\left(0, R_{S}(\cdot)\right)\right)=L^{2}\left(D_{2}, L_{r}^{2}\left(0, R_{S}(\cdot)\right)\right)$.

Since the variables of the model depend on time, then we also introduce the normed spaces $L^{p}(0, t ; X)$, where $1 \leq p \leq \infty$, and $\left(X,\|\cdot\|_{X}\right)$ being a real Banach space.

$L^{p}(0, t ; X):=\left\{v:(0, t) \rightarrow X\right.$ strongly measurable such that $\left.\|v\|_{L^{p}(0, t ; X)}<\infty\right\}$, with $\|v\|_{L^{p}(0, t ; X)}=\left(\int_{0}^{t}\|v(\tau)\|_{X}^{p} d \tau\right)^{1 / p}$ when $1 \leq p<\infty$, and for $p=\infty$, $\|v\|_{L^{\infty}(0, t ; X)}=$ ess $\sup _{0<\tau<t}\|v(\tau)\|_{X}$. Other spaces used in the paper are $W\left(D_{1}\right):=$ $\left\{v \in H^{1}\left(D_{1}\right): \int_{D_{1}} v d x=0\right\}$, which is a closed subspace of $H^{1}\left(D_{1}\right)$ where the potential $\phi_{1}(x, t)$ is calculated, and the space of $q$ times continuously differentiable functions defined on $D, C^{q}(D)$, when $q=0, C^{0}(D):=C(D)$.

Next, we introduce the following regularity assumptions on the data and the molar flux $J[4]$. 
A1)

$$
\begin{gathered}
u^{0} \in H^{1}(D), u^{0}>0, v^{0} \in C\left(\bar{D}_{3}\right), 0<v^{0}<v_{\max }, \\
I(t) \in C_{\text {part }}\left(\left[0, T^{*}\right]\right), 0<T_{\text {end }} \leq T^{*}<\infty,
\end{gathered}
$$

where $C_{\text {part }}$ denotes the set of piecewise continuous functions, i.e.,

$C_{\text {part }}([a, b])=\left\{g:[a, b] \rightarrow \mathbb{R}: \exists a=t_{0}<t_{1}<\cdots t_{N}=b\right.$ such that $\left.g \in C\left(\left[t_{i-1}, t_{i}\right]\right)\right\}$.

A2) For $0<a<c<+\infty, k_{0}$ and $\sigma_{0}$ positive constants,

$$
\begin{gathered}
k_{1} \in L^{\infty}\left(D_{1}\right), k_{1} \geq k_{0}>0, k_{2} \in[a, c], \kappa \in C^{2}((0,+\infty)), \\
\sigma \in L^{\infty}\left(D_{2}\right), \kappa \geq \kappa_{0}>0, \sigma \geq \sigma_{0}>0 .
\end{gathered}
$$

Moreover, $k_{1}:=\inf _{x \in D_{1}} k_{1}(x)$ and $k_{2}:=\min _{x \in D_{2}} k_{2}(x)$.

A3) For all $\left(x, u, v_{s}, \eta\right) \in D_{2} \times(0,+\infty) \times\left(0, v_{s, \max }\right) \times \mathbb{R}$,

$$
J \in C^{2}\left(D_{2} \times(0,+\infty) \times\left(0, v_{s, \max }\right) \times \mathbb{R}\right), \frac{\partial J}{\partial \eta}>0 .
$$

A weak formulation to (5)-(8) is the following. Find

$$
\left\{\begin{array}{l}
u \in L^{2}\left(0, T_{\mathrm{end}} ; H^{1}\left(D_{1}\right)\right), \frac{d u}{d t} \in L^{2}\left(0, T_{\mathrm{end}} ; H^{1}\left(D_{1}\right)\right)^{*}, \\
v \in L^{2}\left(0, T_{\mathrm{end}} ; L^{2}\left(D_{2}, H_{r}^{1}\left(0, R_{S}(\cdot)\right)\right)\right), \frac{d v}{d t} \in L^{2}\left(0, T_{\mathrm{end}} ; L^{2}\left(D_{2}, H_{r}^{1}\left(0, R_{S}(\cdot)\right)\right)\right)^{*}, \\
\phi_{1} \in L^{2}\left(0, T_{\mathrm{end}} ; W\left(D_{1}\right)\right) \text { and } \phi_{2} \in L^{2}\left(0, T_{\mathrm{end}} ; H^{1}\left(D_{2}\right)\right),
\end{array}\right.
$$

such that

$$
\int_{D_{1}} \frac{\partial u}{\partial t} w d x+\int_{D_{1}} k_{1} \frac{\partial u}{\partial x} \frac{d w}{d x} d x=\int_{D_{1}} a_{1} J w d x \forall w \in H^{1}\left(D_{1}\right) ;
$$

for a.e. $x \in D_{2}$ and for all $w \in H_{r}^{1}\left(0, R_{S}(x)\right)$ radially symmetric

$$
\begin{aligned}
& \int_{0}^{R_{s}(x)} \frac{\partial v}{\partial t} w r^{2} d r+\int_{0}^{R_{s}(x)} k_{2} \frac{\partial v}{\partial r} \frac{\partial w}{\partial r} r^{2} d r=\frac{-R_{\mathrm{S}}^{2}(x) J w\left(R_{s}(x)\right)}{a_{2}(x) F} \\
& \int_{D_{1}} \kappa(u) \frac{\partial \phi_{1}}{\partial x} \frac{d w}{d x} d x=\int_{D_{1}} J w d x \forall w \in H^{1}\left(D_{1}\right)
\end{aligned}
$$

and

$$
\int_{D_{2}} \sigma \frac{\partial \phi_{2}}{\partial x} \frac{d w}{d x} d x=-\int_{D_{2}}(J+g) w d x, \forall w \in H^{1}\left(D_{2}\right)
$$


where $L^{2}\left(0, T_{\text {end }} ; H^{1}\left(D_{1}\right)\right)^{*}$ and $L^{2}\left(0, T_{\text {end }} ; L^{2}\left(D_{2}, H_{r}^{1}\left(0, R_{S}(\cdot)\right)\right)\right)^{*}$ denote the respective dual spaces of $L^{2}\left(0, T_{\text {end }} ; H^{1}\left(D_{1}\right)\right)$ and $L^{2}\left(0, T_{\text {end }} ; L^{2}\left(D_{2}, H_{r}^{1}\left(0, R_{S}\right.\right.\right.$ $(\cdot))))$.

Remark 1 Following the arguments of [4], where its non-isothermal P2D model includes an additional time dependent non linear ordinary differential equation for the bulk temperature $T(t)$, one can formulate an alternative definition of the weak solution to (5)-(8) based on its Definition 2.7 and prove, under the assumptions A1A3 and for a partition $t_{0}<t_{1}<\cdots<t_{N}$ of $\left[0, T_{\text {end }}\right], T_{\text {end }}$ being small enough, that there is a unique weak solution $\left(u, v, \phi_{1}, \phi_{2}\right)$ in each interval $\left[t_{n}, t_{n+1}\right]$, such that $\left(u, v, \phi_{1}, \phi_{2}\right) \in C\left(\left[t_{n}, t_{n+1}\right] ; K_{Z}\right)$, where $K_{Z}:=H^{1}\left(D_{1}\right) \times L^{2}\left(D_{2}, H_{r}^{1}\left(0, R_{s}(\cdot)\right)\right) \times$ $W\left(D_{1}\right) \times H^{1}\left(D_{2}\right) .\left(u\left(t_{n}\right), v\left(t_{n}\right)\right)$ being the initial condition in such an interval. Also, Kröner [10] proves a local existence and uniqueness theorem for the weak solution of the isothermal P2D model under less general assumptions than in [4].

\section{The semidiscrete finite element formulation of the isothermal P2D model}

We use $H^{1}$-conforming linear finite elements $\left(P_{1}\right.$-finite elements) for the space approximation of the variables $u(x, t), \phi_{1}(x, t)$ and $\phi_{2}(x, t)$; however, $v(x ; r, t)$ is approximated by nonconforming $P_{0}$-finite elements in the $x$-coordinate and $H^{1}$ conforming $P_{1}$-finite elements in the $r$-coordinate. The family of meshes $D_{1 h}$ constructed on the domain $D_{1}$ includes the points $x=0, x=L_{n}, x=L_{n}+\delta$ and $x=L$ as mesh points; since these points are also boundary points of $D_{2}$, then they are also considered as mesh points in the family of meshes $D_{2 h}$. Figure 2 illustrates the families of meshes that we are going to describe next. Noting that $D_{2} \subset D_{1}$, we choose the family of meshes $D_{2 h}$ as a subset of $D_{1 h}$. Let $N E_{1}$ and $N E_{2}$ be the number of elements of $D_{1 h}$ and $D_{2 h}$ respectively, and let $M_{1}$ and $M_{2}$ be the number of mesh points of such meshes, then, for $i=1,2$, we have that

$$
D_{i h}=\left\{e_{m}\right\}_{m=1}^{N E_{i}} \text { and } \bar{D}_{i}=\cup_{m=1}^{N E_{i}} e_{m}
$$

where the $m$ th element, $e_{m}:=\left\{x: x_{1}^{m} \leq x \leq x_{2}^{m}\right\}$, and $h_{m}:=x_{2}^{m}-x_{1}^{m}$ is the length of the element $e_{m}$; the points $x_{1}^{m} x_{2}^{m}$ are denoted element nodes. We set $h=\max _{m} h_{m}$, and $\gamma=h^{-1} \min _{m} h_{m}$. The parameter $\gamma$ is a measure of the uniformity of the meshes. The collection of all the element nodes defines the set of nodes, $\left\{x_{l}\right\}_{l=1}^{M_{i}}$, of the mesh $D_{i h}$. To construct the family of meshes $\widehat{D}_{3 h \Delta r}$ on $D_{3}$, we recall that $D_{3}=\cup_{x \in D_{2}}\{x\} \times\left(0, R_{S}(x)\right)$, where $R_{S}(x)$ is the radius of the solid spherical particle associated with the point $\{x\}$. Thus, for each mesh point $\left\{x_{l}\right\} \in D_{2 h}$ we define the radial vertical domain $D_{r}^{(l)}:=\left\{r \in \mathbb{R}: 0<r<R_{S}\left(x_{l}\right)\right\}$, which represents the spherical particle at $x_{l}$, and let

$$
D_{\Delta r}^{(l)}=\left\{e_{k}^{(l)}\right\}_{k=1}^{N E^{(l)}} \text { such that } \bar{D}_{r}^{(l)}=\cup_{k=1}^{N E^{(l)}} e_{k}^{(l)}
$$


where $N E^{(l)}$ denotes the number of elements in the interval $\left[0, R_{S}\left(x_{l}\right)\right]$ and $\Delta r_{k}^{(l)}$ is the width of the element $e_{k}^{(l)}:=\left\{r: r_{1}^{(l) k} \leq r \leq r_{2}^{(l) k}\right\}$, we set $\Delta r=\max _{l}\left(\max _{k} \Delta r_{k}^{(l)}\right)$ and $\gamma_{r}=\Delta r^{-1} \min _{l}\left(\min _{k} \Delta r_{k}^{(l)}\right)$. The set of mesh points in each mesh $D_{\Delta r}^{(l)}$ is denoted $\left\{r_{j}^{(l)}\right\}_{j=1}^{M^{(l)}}$. Furthermore, let $\left\{\widehat{e}_{l}\right\}_{l=1}^{M_{2}}$ be the collection of nonconforming elements of the mesh $\widehat{D}_{2 h}$ which are associated with the nodes $\left\{x_{l}\right\}$, they are defined as follows: if $\left\{x_{l}\right\}$ is not a boundary point, then

$$
\widehat{e}_{l}:=\left\{x \in D_{2}: x_{l}-\frac{h_{l-1}}{2} \leq x<x_{l}+\frac{h_{l}}{2}\right\} \text {; }
$$

on the contrary, if $\left\{x_{l}\right\}$ is a left boundary point, then

$$
\widehat{e}_{l}:=\left\{x \in \bar{D}_{2}: x_{l} \leq x<x_{l}+\frac{h_{l}}{2}\right\},
$$

and if $\left\{x_{l}\right\}$ is a right boundary point, then

$$
\widehat{e}_{l}:=\left\{x \in \bar{D}_{2}: x_{l}-\frac{h_{l-1}}{2} \leq x \leq x_{l}\right\} .
$$

We define the meshes $\widehat{D}_{3 h \Delta r}$ as

$$
\widehat{D}_{3 h \Delta r}:=\left\{\widehat{e}_{l} \times \bar{D}_{\Delta r}^{(l)}\right\}_{l=1}^{M_{2}} \text { such that } \bar{D}_{3}:=\cup_{l=1}^{M_{2}} \widehat{e}_{l} \times \bar{D}_{\Delta r}^{(l)}
$$

The families of conforming linear finite element spaces associated with these meshes are the following. For $i=1,2$,

$$
V_{h}^{(1)}\left(\bar{D}_{i}\right):=\left\{v_{h} \in C\left(\bar{D}_{i}\right): \forall e_{m} \in D_{i h},\left.v_{h}\right|_{e_{m}} \in P_{1}\left(e_{m}\right)\right\}
$$

where $P_{1}\left(e_{m}\right)$ denotes the set of linear polynomials defined on $e_{m}$. Let $\left\{\psi_{l}(x)\right\}_{l=1}^{M_{i}}$ be the set of nodal basis functions for the space $V_{h}^{(1)}\left(\bar{D}_{i}\right)$, then any function $v_{h} \in V_{h}^{(1)}\left(\bar{D}_{i}\right)$ can be written as

$$
v_{h}(x)=\sum_{l=1}^{M_{i}} V_{l} \psi_{l}(x), \text { where } V_{l}=v_{h}\left(x_{l}\right) .
$$

Note that $V_{h}^{(1)}\left(\bar{D}_{i}\right) \subset H^{1}\left(D_{i}\right)$. The nonconforming finite element space associated with the mesh $\widehat{D}_{2 h}$ is defined as

$$
V_{h}^{(0)}\left(\bar{D}_{2}\right):=\left\{v_{h} \in L^{2}\left(D_{2}\right): \forall \widehat{e}_{l} \in \widehat{D}_{2 h},\left.v_{h}\right|_{\widehat{e}_{l}} \in P_{0}\left(\widehat{e}_{l}\right)\right\}
$$




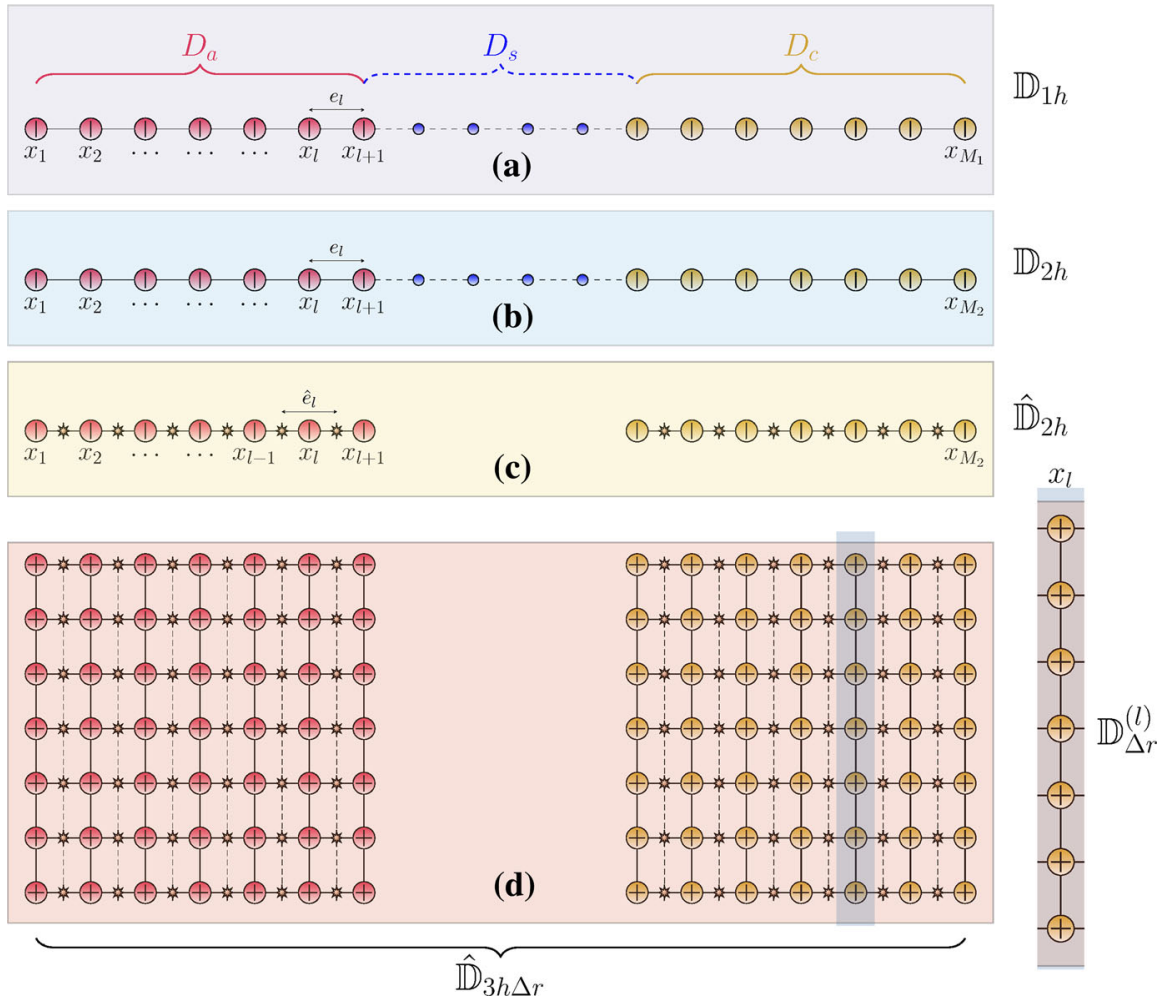

Fig. 2 Panel (a): the mesh for the domain $D_{1}$, which includes the negative electrode (anode in the figure) $D_{a}$, the separator $D_{s}$ and the positive electrode (cathode in the figure) $D_{c}$. Panel $(b)$ : the mesh for the domain $D_{2}=D_{a} \cup D_{c}$. Panel $(c)$ : the mesh of nonconforming elements for the domain $D_{2}$. Panel $(d)$ : the mesh for the domain $D_{3}$

where $P_{0}\left(\widehat{e}_{l}\right)$ is the set of polynomials of degree zero defined on $\widehat{e}_{l}$. Let $\left\{\chi_{l}(x)\right\}_{l=1}^{M_{2}}$ be the set of nodal basis functions for $V_{h}^{(0)}\left(\bar{D}_{2}\right)$,

$$
\chi_{l}(x)=\left\{\begin{array}{l}
1 \text { if } x \in \widehat{e}_{l}, \\
0 \text { otherwise }
\end{array}\right.
$$

then any function $v_{h}(x) \in V_{h}^{(0)}\left(\bar{D}_{2}\right)$ is expressed as

$$
v_{h}(x)=\sum_{l=1}^{M_{2}} V_{l} \chi_{l}(x), \text { where } V_{l}=v_{h}\left(x_{l}\right) .
$$

It is worth remarking that for $1 \leq p<\infty$, the $L^{p}$-norm of $v_{h}(x) \in V_{h}^{(0)}\left(\bar{D}_{2}\right)$ is given as $\left\|v_{h}\right\|_{L^{p}\left(D_{2}\right)}^{p}=\sum_{l=1}^{M_{2}} \widehat{h}_{l} V_{l}^{p}$, where $\widehat{h}_{l}$ denotes the length of the element $\widehat{e}_{l}$, 
and the $L^{2}$-inner product of $v_{h}, w_{h} \in V_{h}^{(0)}\left(\bar{D}_{2}\right), \int_{D_{2}} v_{h} w_{h} d x=\sum_{l=1}^{M_{2}} \widehat{h}_{l} V_{l} W_{l}$. Next, we introduce the finite element space $V_{\Delta r}^{(1)}\left(\bar{D}_{r}^{(l)}\right)$. For $1 \leq l \leq M_{2}$,

$$
V_{\Delta r}^{(1)}\left(\bar{D}_{r}^{(l)}\right):=\left\{v_{\Delta r} \in C\left(\bar{D}_{r}^{(l)}\right): \forall e_{k}^{(l)} \in D_{\Delta r}^{(l)},\left.v_{\Delta r}^{(l)}(r)\right|_{e_{k}^{(l)}} \in P_{1}\left(e_{k}^{(l)}\right)\right\}
$$

So, if $\left\{\alpha_{j}^{(l)}(r)\right\}_{j=1}^{M^{(l)}}$ denotes the set of nodal basis of $V_{\Delta r}\left(\bar{D}_{r}^{(l)}\right)$, any function $v_{\Delta r}^{(l)} \in$ $V_{\Delta r}^{(1)}\left(\bar{D}_{r}^{(l)}\right) \subset H_{r}^{1}\left(0, R_{s}\left(x_{l}\right)\right)$ can be written as

$$
v_{\Delta r}^{(l)}(r)=\sum_{j=1}^{M^{(l)}} V_{j}^{(l)} \alpha_{j}^{(l)}(r), \text { where } V_{j}^{(l)}=v_{h}^{(l)}\left(r_{j}\right)
$$

Regarding the meshes $\widehat{D}_{3 h \Delta r}$, we define the finite element space $V_{h \Delta r}\left(\bar{D}_{3}\right)$ as follows. For $1 \leq l \leq M_{2}$ and $1 \leq k \leq N E^{(l)}$

$V_{h \Delta r}\left(\bar{D}_{3}\right):=\left\{v_{h \Delta r} \in H_{r}^{0,1}\left(D_{2} \times\left(0, R_{s}\right)(\cdot)\right):\left.v_{h \Delta r}(x ; r)\right|_{\widehat{e}_{l} \times e_{k}^{(l)}} \in P_{0}\left(\widehat{e}_{l}\right) \otimes P_{1}\left(e_{k}^{(l)}\right)\right\}$,

noting that when $x \in \widehat{e}_{l}, R_{S}(x)=R_{S}\left(x_{l}\right)$. Hence, any function $v_{h \Delta r}(x ; r) \in V_{h \Delta r}\left(\bar{D}_{3}\right)$ is of the form

$$
v_{h \Delta r}(x ; r)=\sum_{l=1}^{M_{2}} \sum_{j=1}^{M^{(l)}} V_{l j} \chi_{l}(x) \alpha_{j}^{(l)}(r), \text { where } V_{l j}=v_{h \Delta r}\left(x_{l}, r_{j}\right),
$$

or equivalently, using the notation $v_{\Delta r}^{(l)}(r)$ to denote $v_{h \Delta r}\left(x_{l} ; r\right)$, we can write

$$
v_{h \Delta r}(x ; r)=\sum_{l=1}^{M_{2}} v_{\Delta r}^{(l)}(r) \chi_{l}(x)
$$

. The function $v_{s h}(x):=v_{h \Delta r}\left(x ; R_{S}(x)\right)$ is given by the expression

$$
v_{s h}\left(x ; R_{S}(x)\right)=\sum_{l=1}^{M_{2}} V_{l M^{(l)}} \chi_{l}(x),
$$

so that $v_{s h}(x) \in V_{h}^{(0)}\left(\bar{D}_{2}\right)$. We calculate $\phi_{1 h}(x, t)$, which is the approximation to $\phi_{1}(x, t)$, in the finite dimensional space

$$
W_{h}\left(\bar{D}_{1}\right):=\left\{v_{h} \in V_{h}^{(1)}\left(\bar{D}_{1}\right): \int_{D_{1}} v_{h} d x=0\right\}, W_{h}\left(\bar{D}_{1}\right) \subset W\left(D_{1}\right) .
$$


Thus, the finite element formulation is as follows. For all $t \in\left(0, T_{\mathrm{end}}\right)$, the semidiscrete approximation $\left(u_{h}(t), v_{h \Delta r}(t), \phi_{1 h}(t), \phi_{2 h}(t)\right) \in V_{h}^{(1)}\left(\bar{D}_{1}\right) \times V_{h \Delta r}\left(\bar{D}_{3}\right) \times$ $W_{h}\left(\bar{D}_{1}\right) \times V_{h}^{(1)}\left(\bar{D}_{2}\right),\left(u_{h}^{0}, v_{h}^{0}\right) \in V_{h}^{(1)}\left(\bar{D}_{1}\right) \times V_{h \Delta r}\left(\bar{D}_{3}\right)$, is solution to the following system of equations.

$$
\begin{aligned}
& \int_{D_{1}} \frac{\partial u_{h}}{\partial t} w_{h} d x+\int_{D_{1}} k_{1} \frac{\partial u_{h}}{\partial x} \frac{d w_{h}}{d x} d x=\int_{D_{1}} a_{1} J_{h} w_{h} d x \forall w_{h} \in V_{h}^{(1)}\left(\bar{D}_{1}\right) . \\
& \left\{\int_{D_{2}} \int_{0}^{R_{s}(x)} \frac{\partial v_{h \Delta r}}{\partial t} w_{h \Delta r} r^{2} d r d x+\int_{D_{2}} \int_{0}^{R_{s}(x)} k_{2} \frac{\partial v_{h \Delta r}}{\partial r} \frac{\partial w_{h \Delta r}}{\partial r} r^{2} d r d x\right. \\
& =-\int_{D_{2}} \frac{R_{s}^{2}(x) J_{h} w_{h \Delta r}\left(x, R_{s}(x)\right)}{a_{2}(x) F} d x \forall w_{h \Delta r} \in V_{h \Delta r}\left(\bar{D}_{3}\right) . \\
& \int_{D_{1}} \kappa\left(u_{h}\right) \frac{\partial \phi_{1 h}}{\partial x} \frac{d w_{h}}{d x} d x=\int_{D_{1}} J_{h} w_{h} d x \forall w_{h} \in V_{h}^{(1)}\left(\bar{D}_{1}\right) . \\
& \int_{D_{2}} \sigma \frac{\partial \phi_{2 h}}{\partial x} \frac{d w_{h}}{d x} d x=-\int_{D_{2}}\left(J_{h}+g\right) w_{h} d x \forall w_{h} \in V_{h}^{(1)}\left(\bar{D}_{2}\right) . \\
& \int_{D_{1}} J_{h} d x=\int_{D_{2}} J_{h} d x=0, \text { with } \int_{D_{\mathrm{n}}} J_{h} d x=I(t)=-\int_{D_{\mathrm{p}}} J_{h} d x .
\end{aligned}
$$

In this system,

$$
\begin{aligned}
& J_{h}:=J\left(x, u_{h}, v_{s h}, \eta_{h}\right)=a_{2}(x) i_{0 h} \sinh \left(\beta \eta_{h}\right), i_{0 h}:=i_{0}\left(u_{h}, v_{s h}\right) \\
& \eta_{h}:=\phi_{1 h}-\phi_{2 h}-\alpha_{h} \ln u_{h}-\bar{U}_{h}\left(v_{s h}\right), \alpha_{h}=\alpha\left(u_{h}\right) .
\end{aligned}
$$

Note that $J_{h}$ also depends on $t$ through $u_{h}, v_{s h}$ and $\eta_{h}$.

Remark 2 We must note, see (14), that $v_{h \Delta r}$ and $w_{h \Delta r}$ are elementwise constant functions in the $x$ direction, and $J_{h}$ is a piecewise continuous function in $x$ for which it makes sense to consider the approximation, $I_{h}^{(0)} J_{h} \in V_{h}^{(0)}\left(\bar{D}_{2}\right)$, see in Section 4.1 the definition of the interpolant $I_{h}^{(0)}$. Then, approximating $J_{h}$ by $I_{h}^{(0)} J_{h}$ one readily shows, by performing the integral on $D_{2}$, that (16) can be recast as follows: for all mesh-point $\left\{x_{l}\right\} \in D_{2 h}$, calculate $v_{\Delta r}^{(l)}(r, t) \in V_{\Delta r}^{(1)}\left(\bar{D}_{r}^{(l)}\right)$ such that

$$
\begin{gathered}
\int_{0}^{R_{s}\left(x_{l}\right)} \frac{\partial v_{\Delta r}^{(l)}(r, t)}{\partial t} w_{\Delta r}^{(l)}(r) r^{2} d r+\int_{0}^{R_{s}\left(x_{l}\right)} k_{2} \frac{\partial v_{\Delta r}^{(l)}(r, t)}{\partial r} \frac{\partial w_{\Delta r}^{(l)}(r)}{\partial r} r^{2} d r \\
=-R_{s}^{2}\left(x_{l}\right)\left(a_{2}\left(x_{l}\right) F\right)^{-1} J_{h} \mid x=x_{l} w_{\Delta r}^{(l)}\left(R_{s}\left(x_{l}\right)\right) \forall w_{\Delta r}^{(l)} \in V_{\Delta r}^{(1)}\left(\bar{D}_{r}^{(l)}\right) .
\end{gathered}
$$

Once $v_{\Delta r}^{(l)}(r, t)$ is known, one calculates $v_{h \Delta r}$ by the expression (14). 
Notice that this equation is the finite element approximation of (11) for $w \in$ $H_{r}^{1}\left(0, R_{S}\left(x_{l}\right)\right)$, see [1].

Based on Remark 1 and since $H^{1}\left(D_{i}\right) \hookrightarrow C\left(\bar{D}_{i}\right)$, we introduce the following spaces which are used in the error analysis and in the application of the fixed point theorems in Sect. 4.

$$
\begin{aligned}
& S_{P}:=\left\{u \in C_{\text {part }}\left(\bar{D}_{1} \times\left[0, T_{\text {end }}\right]\right): \frac{1}{P} \leq u \leq P\right\}, \\
& S_{Q}:=\left\{w \in C_{\text {part }}\left(\bar{D}_{2} \times\left[0, T_{\text {end }}\right]\right): \frac{1}{Q} \leq w \leq 1-\frac{1}{Q}\right\}, \\
& S_{Q}^{*}:=\left\{w \in C_{\text {part }}^{*}\left(\bar{D}_{2} \times\left[0, T_{\text {end }}\right]\right): \frac{1}{Q} \leq w \leq 1-\frac{1}{Q}\right\},
\end{aligned}
$$

where $P$ and $Q$ are constants sufficiently large; for $i=1,2, C_{\text {part }}\left(\bar{D}_{i} \times\left[0, T_{\text {end }}\right]\right)$ denotes the set of piecewise continuous functions in time and continuous in space and $C_{\text {part }}^{*}\left(\bar{D}_{2} \times\left[0, T_{\text {end }}\right]\right)$ denotes the set of piecewise continuous functions in both time and space. $S_{P}$ is the candidate pool for the concentration $u$ and its approximate $u_{h}$, whereas $S_{Q}$ and $S_{Q}^{*}$ play the same role for the concentrations $\frac{v_{s}}{v_{\max }}$ and $\frac{v_{s h}}{v_{\max }}$ respectively. So, we make the following assumption.

A4) There exist constants $P, Q$ and $K$ sufficiently large such that for almost every $t \in\left[0, T_{\text {end }}\right]$ the following bounds hold:

$$
\frac{1}{P} \leq u(t), u_{h}(t) \leq P, \quad \frac{1}{Q} \leq \frac{v_{s}(t)}{v_{\max }}, \frac{v_{s h}(t)}{v_{\max }} \leq\left(1-\frac{1}{Q}\right),
$$

and for $i=1,2$,

$$
\left\|\frac{\partial \phi_{1}(t)}{\partial x}\right\|_{L^{\infty}\left(D_{1}\right)},\left\|\phi_{i}(t)\right\|_{H^{1}\left(D_{i}\right)},\left\|\phi_{i}(t)\right\|_{L^{\infty}\left(D_{i}\right)},\left\|\phi_{i h}(t)\right\|_{L^{\infty}\left(D_{i}\right)} \leq K .
$$

\section{Error analysis for the semidiscrete problem}

We present in this section the error analysis for the semidiscrete potentials and concentrations. Since the development of such an analysis is long, we have split its presentation in a sequence of three subsections. In the first one, we introduce some auxiliary results needed for the error analysis. The second subsection deals with the error estimate for the potentials. Observing that the P2D model is a nonlinear coupled system of equations, then the error for the potentials depends on the error estimates for the concentrations, the analysis of which is carried out in the last subsection.

\subsection{Auxiliary results}

It is well known [2] that for the finite element spaces $V_{h}^{(p)}\left(\bar{D}_{i}\right)(i=1,2 ; p=0,1)$ the following approximation property holds. For $1 \leq s \leq p+1$, 


$$
\inf _{v_{h} \in V_{h}^{(p)}\left(D_{i}\right)}\left\{\left\|v-v_{h}\right\|_{L^{2}\left(D_{i}\right)}+h\left\|\frac{d^{p}\left(v-v_{h}\right)}{d x^{p}}\right\|_{L^{2}\left(D_{i}\right)}\right\} \leq C h^{s}|v|_{H^{s}\left(D_{i}\right)},
$$

where it should be understood that for $p=0, \frac{d^{p}\left(v-v_{h}\right)}{d x^{p}}=v-v_{h}$. For symmetric radial functions defined in the interval $[0, R]$, let $V_{\Delta r}^{(1)}[0, R]$ be a linear finite element space where we approximate such functions, one can prove, following the approach used to prove Lemmas 1 and 2 in [5], that when $w \in H_{r}^{2}(0, R)$,

$$
\inf _{w_{\Delta r} \in V_{\Delta r}^{(1)}[0, R]}\left\{\left\|w-w_{\Delta r}\right\|_{L_{r}^{2}(0, R)}+\Delta r\left\|\frac{d\left(w-w_{\Delta r}\right)}{d r}\right\|_{L_{r}^{2}(0, R)}\right\} \leq C \Delta r^{2}|w|_{H_{r}^{2}(0, R)} .
$$

We consider the interpolants $I_{h}^{(1)}: C\left(\bar{D}_{i}\right) \rightarrow V_{h}^{(1)}\left(\bar{D}_{i}\right), I_{h}^{(0)}: C\left(\bar{D}_{2}\right) \rightarrow V_{h}^{(0)}\left(\bar{D}_{2}\right)$, and the elliptic projection $P_{1}: H^{1}\left(D_{1}\right) \rightarrow V_{h}^{(1)}\left(D_{1}\right)$ such that for $u \in H^{1}\left(D_{1}\right)$

$$
\int_{D_{1}}\left(k_{1} \frac{d\left(P_{1} u-u\right)}{d x} \frac{d u_{h}}{d x}+\lambda\left(P_{1} u-u\right) u_{h}\right) d x=0 \forall u_{h} \in V_{h}^{(1)}\left(D_{1}\right),
$$

where $\lambda>0$ is a constant; the error analysis for elliptic problems suggests that a good choice is $\lambda=k_{1}$. By virtue of (24) it follows that there exists a constant $C$ independent of $h$ such that

$$
\left\|v-I_{h}^{(0)} v\right\|_{L^{2}\left(D_{2}\right)} \leq C h\|v\|_{H^{1}\left(D_{2}\right)}
$$

for $1 \leq m \leq 2,0 \leq l \leq 1$

$$
\left\|v-I_{h}^{(1)} v\right\|_{H^{l}\left(D_{i}\right)} \leq C h^{m-l}\|v\|_{H^{m}\left(D_{i}\right)}
$$

and from the well known error analysis for elliptic problems [2]

$$
\left\|u-P_{1} u\right\|_{H^{l}\left(D_{1}\right)} \leq C h^{m-l}\|u\|_{H^{m}\left(D_{1}\right)} .
$$

Likewise, for symmetric radial functions $v \in H_{r}^{q}(0, R)$, we define the elliptic projector $P_{1}^{r}: H_{r}^{1}(0, R) \rightarrow V_{\Delta r}^{(1)}[0, R]$ as the solution of the problem

$$
\int_{0}^{R}\left(k_{2} \frac{d\left(P_{1}^{r} w-w\right)}{d r} \frac{d w_{\Delta r}}{d r}+\lambda\left(P_{1}^{r} w-w\right) w_{\Delta r}\right) r^{2} d r=0 \forall w_{\Delta r} \in V_{\Delta r}^{(1)}[0, R],
$$


with $\lambda>0$; as before, a good choice now is $\lambda=k_{2}$. By virtue of (25) it follows that there exists a constant $C$ independent of $\Delta r$ such that

$$
\left\|w-P_{1}^{r} w\right\|_{L_{r}^{2}(0, R)}+\Delta r\left\|\frac{d\left(w-P_{1}^{r} w\right)}{d r}\right\|_{L_{r}^{2}(0, R)} \leq C \Delta r^{2}|w|_{H_{r}^{2}(0, R)} .
$$

$P_{1}^{r}$ can be extended to functions of $x$ and $r$ in an $L^{2}$-sense. Thus, for $(x, r) \in D_{2} \times$ $(0, R(\cdot))$ we define the extended projection $P_{1}^{r}: H_{r}^{1,1}\left(D_{2} \times(0, R(\cdot))\right) \rightarrow L^{2}\left(D_{2}\right) \otimes$ $V_{\Delta r}^{(1)}[0, R(\cdot)]$ as

$$
\begin{aligned}
& \int_{D_{2}} \int_{0}^{R(x)}\left(k_{2} \frac{\partial\left(P_{1}^{r} w-w\right)}{\partial r} \frac{d w_{\Delta r}}{d r}+\lambda\left(P_{1}^{r} w-w\right) w_{\Delta r}\right) r^{2} d r d x \\
& =0 \forall w_{\Delta r} \in L^{2}\left(D_{2}\right) \otimes V_{\Delta r}^{(1)}[0, R(\cdot)] .
\end{aligned}
$$

Assuming that $w \in H_{r}^{1,1}\left(D_{2} \times(0, R(\cdot))\right.$ is such that for a.e. $x \in D_{2}, w(x, \cdot) \in$ $H_{r}^{2}(0, R(x))$, then by virtue of (31)

$$
\begin{aligned}
& \left\|\left(w-P_{1}^{r} w\right)(x, \cdot)\right\|_{L_{r}^{2}(0, R(x))}+\Delta r\left\|\frac{\left(\partial\left(w-P_{1}^{r} w\right)\right)(x, \cdot)}{\partial r}\right\|_{L_{r}^{2}(0, R(x))} \\
& \quad \leq C \Delta r^{2}|w(x, \cdot)|_{H_{r}^{2}(0, R(x))} .
\end{aligned}
$$

Noting that $H_{r}^{0, q}\left(D_{2} \times(0, R(\cdot))\right)=L^{2}\left(D_{2}, L_{r}^{2}(0, R(\cdot))\right) \cap L^{2}\left(D_{2}, H_{r}^{q}(0, R(\cdot))\right)$, then it readily follows that for $q=0,1$

$$
\left\|w-P_{1}^{r} w\right\|_{H_{r}^{0, q}\left(D_{2} \times(0, R(\cdot))\right)} \leq C \Delta r^{2-q}\|w\|_{H_{r}^{0,2}\left(D_{2} \times(0, R(\cdot))\right)} .
$$

We shall also consider the $x$-Lagrange interpolant for functions that depend on $x$ and $r, I_{0}^{x}: H_{r}^{1,1}\left(D_{2} \times(0, R(\cdot))\right) \rightarrow V_{h}^{(0)}\left(\bar{D}_{2}\right) \otimes H_{r}^{1}(0, R(\cdot))$. Thus, for $v(x ; r) \in$ $H_{r}^{1,1}\left(D_{2} \times(0, R(\cdot))\right)$

$$
I_{0}^{x} v(x ; r)=\sum_{l=1}^{M_{2}} v\left(x_{l} ; r\right) \chi_{l}(x) \text { with } r \in\left(0, R\left(x_{l}\right)\right) .
$$

Since $I_{0}^{x}$ can be viewed as an extended Lagrange interpolant $I_{h}^{(0)}: C\left(\bar{D}_{2}\right) \rightarrow$ $V_{h}^{(0)}\left(\bar{D}_{2}\right)$, then based on (27) one can show that

$$
\left\|v-I_{0}^{x} v\right\|_{L^{2}\left(D_{2}, L_{r}^{2}(0, R(\cdot))\right)} \leq C h\|v\|_{H^{1}\left(D_{2} ; L_{r}^{2}\left(0, R_{S}(\cdot)\right)\right)} .
$$


Lemma 3 Let $I=(a, b), 0 \leq a<b$, be a bounded interval with $\bar{I}=[a, b]$, and let $f \in H^{1}(I)$. There exists an arbitrarily small number $\epsilon$ and a positive constant $C(\epsilon)$ such that

$$
\|f\|_{L^{\infty}(I)} \leq \epsilon\left\|\frac{d f}{d x}\right\|_{L^{2}(I)}+C(\epsilon)\|f\|_{L^{2}(I)} .
$$

Proof Since $H^{1}(I) \hookrightarrow C(\bar{I})$, then $f \in C(\bar{I})$ and so does $f^{2}$, so for any $y, z \in I$, $y<z$, we have that

$$
\begin{aligned}
f^{2}(z)-f^{2}(y) & =\int_{y}^{z} \frac{d f^{2}}{d x} d x=2 \int_{y}^{z} f \frac{d f}{d x} d x \\
& \leq \epsilon^{2} \int_{I}\left|\frac{d f}{d x}\right|^{2} d x+\epsilon^{-2} \int_{I} f^{2} d x
\end{aligned}
$$

Since there exists $x^{*} \in I$ such that $f^{2}\left(x^{*}\right)=\min _{x \in I} f^{2}(x)$, then letting $y=x^{*}$ it follows that

$$
f^{2}(y) \leq \frac{1}{|I|} \int_{I} f^{2} d x
$$

Substituting this estimate the result follows.

The next result is a rewording of Lemma 2.4 of [16]. Let $F^{1}(R)$ be the closure of $C^{\infty}$ - functions with respect to the $H_{r}^{1}(0, R)$-norm and with the property that their with first derivative vanishes at $r=0$.

Lemma 4 If $v \in F^{1}(R)$, then for all $0<a<R$,

1) $v \in H^{1}(a, R)$.

2) There exists an arbitrarily small number $\epsilon$ and a positive (possibly large) constant $C(\epsilon)$, both depending on a, such that

$$
\|v\|_{L^{\infty}(a, R)} \leq \epsilon\left\|\frac{d v}{d r}\right\|_{L_{r}^{2}(0, R)}+C(\epsilon)\|v\|_{L_{r}^{2}(0, R)} .
$$

Proof To prove 1) we note that for any $v \in F^{1}(R)$,

$$
\begin{aligned}
& \|v\|_{H_{r}^{1}(0, R)}^{2}=\int_{0}^{R} v^{2} r^{2} d r+\int_{0}^{R}\left|\frac{d v}{d r}\right|^{2} r^{2} d r \\
& \geq \int_{a}^{R} v^{2} r^{2} d r+\int_{a}^{R}\left|\frac{d v}{d r}\right|^{2} r^{2} d r \\
& \geq a^{2} \int_{a}^{R} v^{2} d r+\int_{a}^{R}\left|\frac{d v}{d r}\right|^{2} d r=a^{2}\|v\|_{H^{1}(a, R)}^{2} .
\end{aligned}
$$


So, any sequence $\left\{v_{n}\right\}$ that converges with respect to the $H_{r}^{1}(0, R)$-norm also converges with respect to the $H^{1}(a, R)$-norm. As for the point 2$)$, we notice that from (36) and (38) it readily follows (37).

Lemma 5 For each $(x, t) \in D_{2} \times\left[0, T_{\text {end }}\right]$ we have the following estimates.

$$
\begin{aligned}
\left|i_{0}-i_{0 h}\right| & \leq C\left|u-u_{h}\right|+C\left|v_{s}-v_{h s}\right| \\
\left|\ln u-\ln u_{h}\right| & \leq C\left|u-u_{h}\right| \\
\left|\bar{U}\left(v_{s}\right)-\bar{U}\left(v_{s h}\right)\right| & \leq C\left|v_{s}-v_{s h}\right|
\end{aligned}
$$

Proof Noting that the functions $x \rightarrow \sqrt{x}, x \rightarrow \sqrt{1-x}, x \rightarrow \ln x$ and $x \rightarrow \bar{U}(x)$ are smooth bounded and Lipschitz functions in any bounded interval $[a, b], 0<a<b$, and that the composition and multiplication of bounded Lipschitz functions results in a Lipschitz function, then the estimates follow. The constant $C$ in (39) depends on the constants $P$ and $Q$ of (22).

Lemma 6 Let us consider $J$ and its approximate $J_{h}$, then for a.e. $t \in\left[0, T_{\text {end }}\right]$ there exists a positive constant $C$ such that

$$
\begin{aligned}
& \left\|J-J_{h}\right\|_{L^{2}\left(D_{2}\right)}^{2} \leq C\left\{\left\|\phi_{2}(t)-\phi_{2 h}(t)\right\|_{L^{2}\left(D_{2}\right)}^{2}+\left\|\phi_{1}(t)-\phi_{1 h}(t)\right\|_{L^{2}\left(D_{1}\right)}^{2}\right. \\
& \left.+\left\|u(t)-u_{h}(t)\right\|_{L^{2}\left(D_{1}\right)}^{2}+\left\|v_{s}(t)-v_{s h}(t)\right\|_{L^{2}\left(D_{2}\right)}^{2}\right\}
\end{aligned}
$$

Proof Recalling the expressions for $J$, see (2)-(3), and $J_{h}$, see (20), using the assumption $\mathbf{A} 4$ and the bounds (22) and (23), we have that for all $(x, t) \in D_{2} \times\left[0, T_{\text {end }}\right]$

$$
\begin{aligned}
\left|J-J_{h}\right| & =\left|a_{2} i_{0} \sinh (\beta \eta)-a_{2} i_{0 h} \sinh \left(\beta \eta_{h}\right)\right| \\
& \leq\left|a_{2} i_{0}\left(\sinh (\beta \eta)-\sinh \left(\beta \eta_{h}\right)\right)\right|+\left|a_{2}\left(i_{0}-i_{0 h}\right) \sinh \left(\beta \eta_{h}\right)\right| \\
& \leq C\left|\sinh (\beta \eta)-\sinh \left(\beta \eta_{h}\right)\right|+\left\|a_{2} \sinh \left(\beta \eta_{h}\right)\right\|_{L^{\infty}\left(D_{2} \times\left[0, T_{\text {end }}\right]\right)}\left|i_{0}-i_{0 h}\right| \\
& \leq C\left|\sinh (\beta \eta)-\sinh \left(\beta \eta_{h}\right)\right|+C\left|i_{0}-i_{0 h}\right| .
\end{aligned}
$$

where due to the bounds (22) and (23) the constants $C=C(P, Q, K)$. Now, by virtue of the mean value theorem there exists $z \in\left(\eta, \eta_{h}\right)$ such that

$$
\left|\sinh (\beta \eta)-\sinh \left(\beta \eta_{h}\right)\right| \leq \beta|\cosh (z)|\left|\eta-\eta_{h}\right| \leq C\left|\eta-\eta_{h}\right|
$$

and resorting again to (22) and (23) it follows that

$$
\|z\|_{L^{\infty}\left(D_{2} \times\left[0, T_{\text {end }}\right]\right)} \leq\|\eta\|_{L^{\infty}\left(D_{2} \times\left[0, T_{\text {end }}\right]\right)}+\left\|\eta_{h}\right\|_{L^{\infty}\left(D_{2} \times\left[0, T_{\text {end }}\right]\right)} \leq C
$$

Hence, applying Lemma 5 yields

$$
\left|J-J_{h}\right| \leq C\left(\left|\phi_{2}-\phi_{2 h}\right|+\left|\phi_{1}-\phi_{1 h}\right|+\left|u-u_{h}\right|+\left|v_{s}-v_{s h}\right|\right)
$$

From this estimate it follows (40). 


\subsection{Error estimates for the potentials}

To estimate the error for the potentials $\phi_{1}$ and $\phi_{2}$ is convenient to introduce the spaces $V:=H^{1}\left(D_{1}\right) \times H^{1}\left(D_{2}\right):=\left\{w=\left(w_{1}, w_{2}\right): w_{1} \in H^{1}\left(D_{1}\right), w_{2} \in H^{1}\left(D_{2}\right)\right\}$ and $V_{h} \subset V$, where $V_{h}:=V_{h}^{(1)}\left(\bar{D}_{1}\right) \times V_{h}^{(1)}\left(\bar{D}_{2}\right) . V$ is a Hilbert space with norm

$$
\|w\|_{V}=\left(\left\|w_{1}\right\|_{H^{1}\left(D_{1}\right)}^{2}+\left\|w_{2}\right\|_{H^{1}\left(D_{2}\right)}^{2}\right)^{1 / 2}
$$

and seminorm

$$
|v|_{V}=\left(\left|w_{1}\right|_{H^{1}\left(D_{1}\right)}^{2}+\left|w_{2}\right|_{H^{1}\left(D_{2}\right)}^{2}\right)^{1 / 2}
$$

Considering the bilinear forms $a_{1}: H^{1}\left(D_{1}\right) \times H^{1}\left(D_{1}\right) \rightarrow \mathbb{R}$ and $a_{2}: H^{1}\left(D_{2}\right) \times$ $H^{1}\left(D_{2}\right) \rightarrow \mathbb{R}$

$$
\left\{\begin{array}{l}
a_{1}\left(\phi_{1}, \psi_{1}\right)=\int_{D_{1}} \kappa(u) \frac{d \phi_{1}}{d x} \frac{d \psi_{1}}{d x} d x, \\
a_{2}\left(\phi_{2}, \psi_{2}\right)=\int_{D_{2}} \sigma \frac{d \phi_{2}}{d x} \frac{d \psi_{2}}{d x} d x
\end{array}\right.
$$

we can define the bilinear form $a: V \times V \rightarrow \mathbb{R}$ as follows. Let $\Phi$ and $\Psi \in V$, $\Phi=\left(\phi_{1}, \phi_{2}\right)$ and $\Psi=\left(\psi_{1}, \psi_{2}\right)$, then

$$
a(\Phi, \Psi)=a_{1}\left(\phi_{1}, \psi_{1}\right)+a_{2}\left(\phi_{2}, \psi_{2}\right)
$$

Furthermore, concerning the right hand side terms of (12) and (13), we introduce the operator $B: V \rightarrow V^{*}, V^{*}$ being the dual for $V$, as

$$
\langle B(\Phi), \Psi\rangle=\int_{D_{2}} J\left(\psi_{2}-\psi_{1}\right) d x .
$$

Hence, we can recast the equations (12) and (13) as follows. Find $\Phi \in L^{2}\left(0, T_{\text {end }}\right.$; $\left.W\left(D_{1}\right) \times H^{1}\left(D_{2}\right)\right)$ such that

$$
a(\Phi, \Psi)+\langle B(\Phi), \Psi\rangle=-\int_{D_{2}} g \psi_{2} d x \quad \forall \Psi \in V .
$$

Likewise, the finite element solutions $\phi_{1 h}$ and $\phi_{2 h}$ that satisfy (17) and (18) respectively, can be formulated as follows. For all $t \in\left[0, T_{\text {end }}\right]$, find $\Phi_{h} \in W_{h}\left(\bar{D}_{1}\right) \times$ $V_{h}^{(1)}\left(\bar{D}_{2}\right)$ such that

$$
a_{h}\left(\Phi_{h}, \Psi_{h}\right)+\left\langle B_{h}\left(\Phi_{h}\right), \Psi_{h}\right\rangle=-\int_{D_{2}} g \psi_{2 h} d x \forall \Psi_{h} \in V_{h}
$$


where

$$
a_{h}\left(\Phi_{h}, \Psi_{h}\right)=a_{1 h}\left(\phi_{1 h}, \psi_{1 h}\right)+a_{2}\left(\phi_{2 h}, \psi_{2 h}\right),
$$

with

$$
a_{1 h}\left(\phi_{1 h}, \psi_{1 h}\right)=\int_{D_{1}} \kappa\left(u_{h}\right) \frac{d \phi_{1 h}}{d x} \frac{d \psi_{1 h}}{d x} d x
$$

and

$$
\left\langle B_{h}\left(\Phi_{h}\right), \Psi_{h}\right\rangle=\int_{D_{2}} J_{h}\left(\psi_{2 h}-\psi_{1 h}\right) d x
$$

Remark 7 The existence and uniqueness of $\Phi$ is proven in [4] under the same kind of assumptions as A1-A4, whereas in [19] and [10] the existence is proven applying Schauder Fixed Point Theorem [7] and the uniqueness using the fact that $\phi_{1}(x) \in$ $W\left(D_{1}\right)$.

As for the bilinear form $a$ and the operator $B$, we have the following result.

Lemma 8 Assuming that A1-A4 hold, we have that: (i) the bilinear form a is continuous, (ii) the operator $B$ is monotone, i.e.,

$$
\langle B(\Phi)-B(\widehat{\Phi}), \Phi-\widehat{\Phi}\rangle \geq 0
$$

bounded and continuous in the sense that for all $\Psi \in V$ there exists a constant $C$ such that

$$
\langle B(\Phi)-B(\widehat{\Phi}), \Psi\rangle \leq C\left(\|\Phi-\widehat{\Phi}\|_{V}\right)\left(\left\|\psi_{1}\right\|_{L^{2}\left(D_{1}\right)}+\left\|\psi_{2}\right\|_{L^{2}\left(D_{2}\right)}\right)
$$

Proof It is easy to prove the continuity of the bilinear form $a$ if one takes into account the regularity assumption A2. To prove (45) we note that

$$
\langle B(\Phi)-B(\widehat{\Phi}), \Phi-\widehat{\Phi}\rangle=\int_{D_{2}} a_{2} i_{0}(\sinh (\beta \eta)-\sinh (\beta \widehat{\eta}))\left(\left(\phi_{2}-\widehat{\phi}_{2}\right)-\left(\phi_{1}-\widehat{\phi}_{1}\right)\right) d x
$$

where $\eta=\phi_{2}-\phi_{1}-\alpha \ln u-\bar{U}$ and $\widehat{\eta}=\widehat{\phi}_{2}-\widehat{\phi}_{1}-\alpha \ln u-\bar{U}$. Since for all $x \in D_{2}$ $a_{2} i_{0}>0$, then by virtue of $\mathbf{A} \mathbf{4}$ we can choose a constant $C(P, Q)$ such that for all $x \in D_{2} C(P, Q) \leq a_{2} i_{0}$, and by the mean value theorem $\sinh (\beta \eta)-\sinh (\beta \widehat{\eta}) \geq$ $\beta(\eta-\widehat{\eta})$, then one readily obtains

$$
\langle B(\Phi)-B(\widehat{\Phi}), \Phi-\widehat{\Phi}\rangle \geq C \int_{D_{2}}\left(\left(\phi_{2}-\phi_{1}\right)-\left(\widehat{\phi}_{2}-\widehat{\phi}_{1}\right)\right)^{2} d x \geq 0
$$


To prove that $B$ is bounded we notice that for all $\Phi \in V$

$$
\langle B(\Phi), \Phi\rangle \leq \int_{D_{2}}|J| \times\left|\phi_{2}-\phi_{1}\right| d x=\int_{D_{2}} \mid a_{2} i_{0}\left(\sinh (\beta \eta)|\times| \phi_{2}-\phi_{1} \mid d x,\right.
$$

but $|J|$ is bounded by virtue of $\mathbf{A 4}$, then using the Cauchy-Schwarz inequality it readily follows that there exists a bounded positive constant $C$ such that

$$
\langle B(\Phi), \Phi\rangle \leq C\|\Phi\|_{V}
$$

so $B$ is bounded. To prove that $B$ is continuous, we again notice that

$$
\langle B(\Phi)-B(\widehat{\Phi}), \Psi\rangle \leq \int_{D_{2}}\left|a_{2} i_{0}(\sinh (\beta \eta)-\sinh (\beta \widehat{\eta}))\right| \times\left|\psi_{2}-\psi_{1}\right| d x,
$$

so, arguing as in the proof of Lemma 6 we have that there exists a positive constant $C$ such that

$$
\left|a_{2} i_{0}(\sinh (\beta \eta)-\sinh (\beta \widehat{\eta}))\right| \leq C|\eta-\widehat{\eta}|=C\left|\left(\phi_{2}-\widehat{\phi}_{2}\right)-\left(\phi_{1}-\widehat{\phi}_{1}\right)\right|
$$

Substituting this estimate in the above inequality and making use of the CauchySchwarz inequality it follows that

$$
\begin{aligned}
& \langle B(\Phi)-B(\widehat{\Phi}), \Psi\rangle \leq C\left(\left\|\phi_{2}-\widehat{\phi}_{2}\right\|_{L^{2}\left(D_{2}\right)}+\left\|\phi_{1}-\widehat{\phi}_{1}\right\|_{L^{2}\left(D_{1}\right)}\right) \\
& \quad\left(\left\|\psi_{2}\right\|_{L^{2}\left(D_{2}\right)}+\left\|\psi_{1}\right\|_{L^{2}(D 1)}\right) \leq C\|\Phi-\widehat{\Phi}\|_{V}\left(\left\|\psi_{2}\right\|_{L^{2}\left(D_{2}\right)}+\left\|\psi_{1}\right\|_{L^{2}(D 1)}\right) .
\end{aligned}
$$

Corollary 9 The discrete bilinear form $a_{h}$ defined in (43) is continuous. The discrete operator $B_{h}$ defined in (44) is monotone, bounded and continuous.

Theorem 10 For a.e. $t \in\left[0, T_{\mathrm{end}}\right]$, let the solution of $(41), \Phi(t)=\left(\phi_{1}(t), \phi_{2}(t)\right)$, be in $H^{2}\left(D_{1}\right) \times H^{2}\left(D_{2}\right)$. There exists a constant $C$ independent of $h$ such that

$$
\begin{aligned}
\left\|\Phi(t)-\Phi_{h}(t)\right\|_{V}^{2} \leq & C\left(h^{2}\left(\left\|\phi_{1}(t)\right\|_{H^{2}\left(D_{1}\right)}^{2}+\left\|\phi_{2}(t)\right\|_{H^{2}\left(D_{2}\right)}^{2}\right)\right. \\
& \left.+\left\|u(t)-u_{h}(t)\right\|_{L^{2}\left(D_{1}\right)}^{2}+\left\|v_{s}(t)-v_{s h}(t)\right\|_{L^{2}\left(D_{2}\right)}^{2}\right) .
\end{aligned}
$$

Proof Setting $\Psi=\Psi_{h}$ in (41) and subtracting (42) yields

$$
a\left(\Phi, \Psi_{h}\right)-a_{h}\left(\Phi_{h}, \Psi_{h}\right)+\left\langle B(\Phi)-B\left(\Phi_{h}\right), \Psi_{h}\right\rangle=-\left\langle B\left(\Phi_{h}\right)-B_{h}\left(\Phi_{h}\right), \Psi_{h}\right\rangle .
$$

Noting that

$$
a\left(\Phi, \Psi_{h}\right)-a_{h}\left(\Phi_{h}, \Psi_{h}\right)=a_{h}\left(\Phi-\Phi_{h}, \Psi_{h}\right)+\int_{D_{1}}\left(\kappa(u)-\kappa\left(u_{h}\right)\right) \frac{\partial \phi_{1}}{\partial x} \frac{d \psi_{1 h}}{d x} d x
$$


it follows that

$$
\begin{aligned}
& a_{h}\left(\Phi-\Phi_{h}, \Psi_{h}\right)+\left\langle B(\Phi)-B\left(\Phi_{h}\right), \Psi_{h}\right\rangle=\left\langle B_{h}\left(\Phi_{h}\right)-B\left(\Phi_{h}\right), \Psi_{h}\right\rangle \\
& \quad-\int_{D_{1}}\left(\kappa(u)-\kappa\left(u_{h}\right)\right) \frac{\partial \phi_{1}}{\partial x} \frac{d \psi_{1 h}}{d x} d x .
\end{aligned}
$$

To estimate the terms of this expression we choose $\Psi_{h}=I_{h}^{(1)} \Phi-\Phi_{h}=$ $\left(I_{h}^{(1)} \phi_{1}-\phi_{1 h}, I_{h}^{(1)} \phi_{2}-\phi_{2 h}\right), I_{h}^{(1)}$ being the Lagrange interpolant on $V_{h}=V_{h}^{(1)}\left(\bar{D}_{1}\right) \times$ $V_{h}^{(1)}\left(\bar{D}_{2}\right)$, this means that $I_{h}^{(1)} \phi_{1} \in V_{h}^{(1)}\left(\bar{D}_{1}\right)$ and $I_{h}^{(1)} \phi_{2} \in V_{h}^{(1)}\left(\bar{D}_{2}\right)$. For convenience, we shall split the expression for $\Psi_{h}$ as

$$
\Psi_{h}=\left(\Phi-\Phi_{h}\right)+\left(I_{h}^{(1)} \Phi-\Phi\right)
$$

Replacing this expression for $\Psi_{h}$ in (49) we have that

$$
\left\{\begin{array}{l}
a_{h}\left(\Phi-\Phi_{h}, \Phi-\Phi_{h}\right)+\left\langle B(\Phi)-B\left(\Phi_{h}\right), \Phi-\Phi_{h}\right\rangle \\
=a_{h}\left(\Phi-\Phi_{h}, \Phi-I_{h}^{(1)} \Phi\right)+\left\langle B(\Phi)-B\left(\Phi_{h}\right), \Phi-I_{h}^{(1)} \Phi\right\rangle \\
+\left\langle B_{h}\left(\Phi_{h}\right)-B\left(\Phi_{h}\right),\left(\Phi-\Phi_{h}\right)+\left(I_{h}^{(1)} \Phi-\Phi\right)\right\rangle \\
+\int_{D_{1}}\left(\kappa(u)-\kappa\left(u_{h}\right)\right) \frac{\partial \phi_{1}}{\partial x} \frac{\partial\left(\left(\phi_{1}-\phi_{1 h}\right)+\left(I_{h}^{(1)} \phi_{1}-\phi_{1 h}\right)\right)}{\partial x} d x
\end{array}\right.
$$

We bound the terms of (50). We start by showing that there exists a positive constant $\alpha$ such that the term on the left hand side satisfies

$$
a_{h}\left(\Phi-\Phi_{h}, \Phi-\Phi_{h}\right)+\left\langle B(\Phi)-B\left(\Phi_{h}\right), \Phi-\Phi_{h}\right\rangle \geq \alpha\left\|\Phi-\Phi_{h}\right\|_{V}^{2} .
$$

To do so we note that by virtue of (47)

$$
\begin{aligned}
& a_{h}\left(\Phi-\Phi_{h}, \Phi-\Phi_{h}\right)+\left\langle B(\Phi)-B\left(\Phi_{h}\right), \Phi-\Phi_{h}\right\rangle \\
& \geq a_{1 h}\left(\phi_{1}-\phi_{1 h}, \phi_{1}-\phi_{1 h}\right)+a_{2}\left(\phi_{2}-\phi_{2 h}, \phi_{2}-\phi_{2 h}\right) \\
& \quad+C \int_{D_{2}}\left(\left(\phi_{2}-\phi_{1}\right)-\left(\phi_{2 h}-\phi_{1 h}\right)\right)^{2} d x .
\end{aligned}
$$

Since $\phi_{1}-\phi_{1 h} \in W\left(D_{1}\right)$, we can use $\mathbf{A} 2$ and Poincaré-Wirtinger inequality to bound $a_{1}\left(\phi_{1}-\phi_{1 h}, \phi_{1}-\phi_{1 h}\right)$ from below as

$$
a_{1 h}\left(\phi_{1}-\phi_{1 h}, \phi_{1}-\phi_{1 h}\right) \geq c_{1}\left\|\phi_{1}-\phi_{1 h}\right\|_{H^{1}\left(D_{1}\right)}^{2},
$$


where the constant $c_{1}=\kappa_{0}\left(1+C_{P}\right)^{-1}, C_{P}$ being the constant of the PoincaréWirtinger inequality; using again $\mathbf{A} 2$, we bound the term $a_{2}\left(\phi_{2}-\phi_{2 h}, \phi_{2}-\phi_{2 h}\right)$ as

$$
a_{2}\left(\phi_{2}-\phi_{2 h}, \phi_{2}-\phi_{2 h}\right) \geq \sigma_{0}\left|\phi_{2}-\phi_{2 h}\right|_{H^{1}\left(D_{2}\right)}^{2} .
$$

Applying Young inequality we find that there exists a constant $\gamma \in(0,1)$ such that

$$
\begin{aligned}
& C \int_{D_{2}}\left(\left(\phi_{2}-\phi_{1}\right)-\left(\phi_{2 h}-\phi_{1 h}\right)\right)^{2} d x . \\
& \geq C\left[\left(1-\frac{4}{\gamma}\right)\left\|\phi_{1}-\phi_{1 h}\right\|_{L^{2}\left(D_{2}\right)}^{2}+(1-\gamma)\left\|\phi_{2}-\phi_{2 h}\right\|_{L^{2}\left(D_{2}\right)}^{2}\right] \\
& \geq C\left[\left(1-\frac{4}{\gamma}\right)\left\|\phi_{1}-\phi_{1 h}\right\|_{L^{2}\left(D_{1}\right)}^{2}+(1-\gamma)\left\|\phi_{2}-\phi_{2 h}\right\|_{L^{2}\left(D_{2}\right)}^{2}\right] .
\end{aligned}
$$

Now, we can choose the constants $C$ and $\gamma$ such that $c_{2}=c_{1}+C\left(1-\frac{4}{\gamma}\right)>0$, and substitute these bounds in (52) to obtain the inequality (51), where $\alpha=$ $\min \left(c_{1}, c_{2}, \sigma_{0},(1-\gamma) C\right)$. Next, we bound the terms on the right hand side. By continuity of the bilinear form and Young inequality, we find that there exists a small positive number $\epsilon_{1}$ and a constant $C\left(\epsilon_{1}\right)$ such that

$$
\begin{aligned}
a_{h}\left(\Phi-\Phi_{h}, \Phi-I_{h}^{(1)} \Phi\right) & \leq C\left\|\Phi-\Phi_{h}\right\|_{V}\left\|\Phi-I_{h}^{(1)} \Phi\right\|_{V} \\
& \leq \epsilon_{1}\left\|\Phi-\Phi_{h}\right\|_{V}^{2}+C\left(\epsilon_{1}\right)\left\|\Phi-I_{h}^{(1)} \Phi\right\|_{V}^{2}
\end{aligned}
$$

To bound $\left\langle B(\Phi)-B\left(\Phi_{h}\right), \Phi-I_{h}^{(1)} \Phi\right\rangle$ we note that

$$
\left\langle B(\Phi)-B\left(\Phi_{h}\right), \Phi-I_{h}^{(1)} \Phi\right\rangle \leq \int_{D_{2}}\left|a_{2} i_{0}\right|\left|\int_{\beta \eta_{h}}^{\beta \eta} \cosh \xi d \xi\right|\left|\Phi-I_{h}^{(1)} \Phi\right| d x
$$

By virtue of (22) and (23) and the mean value theorem for the integral

$$
\begin{aligned}
\left\langle B(\Phi)-B\left(\Phi_{h}\right), \Phi-I_{h}^{(1)} \Phi\right\rangle \leq & C \int_{D_{2}}\left|\eta-\eta_{h}\right|\left|\Phi-I_{h}^{(1)} \Phi\right| d x \\
\leq & C \int_{D_{2}}\left(\left|\phi_{2}-\phi_{2 h}\right|+\left|\phi_{1}-\phi_{1 h}\right|\right. \\
& \left.+\left|u-u_{h}\right|+\left|v_{s}-v_{s h}\right|\right)\left|\Phi-I_{h}^{(1)} \Phi\right| d x .
\end{aligned}
$$


Applying Young inequality yields

$$
\begin{aligned}
\left\langle B(\Phi)-B\left(\Phi_{h}\right), \Phi-I_{h}^{(1)} \Phi\right\rangle \leq & \epsilon_{2}\left(\left\|\Phi-\Phi_{h}\right\|_{V}^{2}+\left\|u-u_{h}\right\|_{L^{2}\left(D_{1}\right)}^{2}\right. \\
& \left.+\left\|v_{s}-v_{s h}\right\|_{L^{2}\left(D_{2}\right)}^{2}\right)+C\left(\epsilon_{2}\right)\left\|\Phi-I_{h}^{(1)} \Phi\right\|_{V}^{2}
\end{aligned}
$$

where $\epsilon_{2}$ is a small positive number and $C\left(\epsilon_{2}\right)$ is a constant. Next, noting that by virtue of (22) and (23) $\left|\sinh \left(\beta \eta_{h}\right)\right|$ is bounded in $D_{2}$, then

$$
\begin{aligned}
& \left\langle B_{h}\left(\Phi_{h}\right)-B\left(\Phi_{h}\right),\left(\Phi-\Phi_{h}\right)+\left(I_{h}^{(1)} \Phi-\Phi\right)\right\rangle \\
& \quad \leq C \int_{D_{2}} a_{2}\left|i_{0}-i_{0 h}\right|\left|\left(\Phi-\Phi_{h}\right)+\left(I_{h}^{(1)} \Phi-\Phi\right)\right| d x .
\end{aligned}
$$

Again, using Lemma 5 and Young inequality we obtain that there exist a small number $\epsilon_{3}$ and a constant $C\left(\epsilon_{3}\right)$ such that

$$
\begin{gathered}
\left\langle B_{h}\left(\Phi_{h}\right)-B\left(\Phi_{h}\right),\left(\Phi-\Phi_{h}\right)+\left(I_{h}^{(1)} \Phi-\Phi\right)\right\rangle \leq \epsilon_{3}\left\|\Phi-\Phi_{h}\right\|_{V}^{2} \\
\quad+\epsilon_{3}\left(\left\|u-u_{h}\right\|_{L^{2}\left(D_{1}\right)}^{2}+\left\|v_{s}-v_{s h}\right\|_{L^{2}\left(D_{2}\right)}^{2}\right)+C\left(\epsilon_{3}\right)\left\|\Phi-I_{h}^{(1)} \Phi\right\|_{V}^{2}
\end{gathered}
$$

To bound the last term on the right hand side of (50) we note that $\left\|\frac{\partial \phi_{1}}{\partial x}\right\|_{L^{\infty}\left(D_{1}\right)}$ is bounded and by virtue of assumption $\mathbf{A 4}$, and by the mean value theorem, $\left|\left(\kappa(u)-\kappa\left(u_{h}\right)\right)\right| \leq C\left|u-u_{h}\right|$, then it follows that

$$
\begin{aligned}
& \int_{D_{1}}\left(\kappa(u)-\kappa\left(u_{h}\right)\right) \frac{\partial \phi_{1}}{\partial x} \frac{\partial\left(\left(\phi_{1}-\phi_{1 h}\right)+\left(I_{h}^{(1)} \phi_{1}-\phi_{1 h}\right)\right)}{\partial x} d x \\
& \leq C \int_{D_{1}}\left|u-u_{h}\right|\left|\frac{\partial\left(\left(\phi_{1}-\phi_{1 h}\right)+\left(I_{h}^{(1)} \phi_{1}-\phi_{1 h}\right)\right)}{\partial x}\right| d x \\
& \leq \epsilon_{4}\left\|\Phi-\Phi_{h}\right\|_{V}^{2}+C\left(\epsilon_{4}\right)\left(\left\|u-u_{h}\right\|_{L^{2}\left(D_{1}\right)}^{2}+\left\|\Phi-I_{h}^{(1)} \Phi\right\|_{V}^{2}\right) .
\end{aligned}
$$

Letting $\epsilon_{1}+\cdots+\epsilon_{4}=\alpha / 2$ and noting that, see (34),

$$
\begin{aligned}
\left\|\Phi-I_{h}^{(1)} \Phi\right\|_{V}^{2} & =\left\|\phi_{1}-I_{h}^{(1)} \phi_{1}\right\|_{H^{1}\left(D_{1}\right)}^{2}+\left\|\phi_{2}-I_{h}^{(1)} \phi_{2}\right\|_{H^{1}\left(D_{2}\right)}^{2} \\
& \leq C h^{2}\left(\left\|\phi_{1}\right\|_{H^{2}\left(D_{1}\right)}^{2}+\left\|\phi_{2}\right\|_{H^{2}\left(D_{2}\right)}^{2}\right)
\end{aligned}
$$

the estimate (48) follows from (50)-(56). 


\subsection{Error estimates for the concentrations $u(x, t)$ and $v(x ; r, t)$}

We wish to estimate $u(x, t)-u_{h}(x, t)$ and $v(x ; r, t)-v_{h \Delta r}(x ; r, t)$ in the $L^{2}-$ norm assuming that both $u(x, t)$ and $v(x ; r, t)$ are as regular as required. Following the standard approach, we decompose $u-u_{h}$ as

$$
u-u_{h}=\left(u-P_{1} u\right)+\left(P_{1} u-u_{h}\right) \equiv \rho_{u}+\theta_{u},
$$

where $P_{1}$ is the elliptic projector defined in (26), and note that $\theta_{u}(x, t) \in V_{h}^{(1)}\left(\bar{D}_{1}\right)$. To carry out a decomposition of this kind for $v(x ; r, t)-v_{h \Delta r}(x ; r, t)$, at first we can try using the extended elliptic projector $P_{1}^{r}: H_{r}^{1,1}\left(D_{2} \times\left(0, R_{S}(\cdot)\right)\right) \rightarrow L^{2}\left(D_{2}\right) \otimes$ $V_{\Delta r}^{(1)}\left[0, R_{S}(\cdot)\right]$ defined in (32) and assume that for all $t, v(x ; r, t) \in H_{r}^{1,1}\left(D_{2} \times\right.$ $\left.\left(0, R_{S}(\cdot)\right)\right)$, then we find that for a. e. $x \in D_{2}$

$$
P_{1}^{r} v(x ; r, t)=\sum_{j=1}^{M} P_{1}^{r} v\left(x ; r_{j}, t\right) \alpha_{j}(r)
$$

here, $M$ denotes the number of mesh points in $\left[0, R_{S}(\cdot)\right],\left\{\alpha_{j}(r)\right\}_{j=1}^{M}$ the nodal basis of the linear finite element space $V_{\Delta r}^{(1)}\left[0, R_{S}(\cdot)\right] \subset H_{r}^{1}\left(0, R_{S}(\cdot)\right)$ and the function $P_{1}^{r} v\left(x ; r_{j}, t\right) \in L^{2}\left(D_{2}\right)$; so, in general, $P_{1}^{r} v(x ; r, t)$ is not in $V_{h \Delta r}\left(\bar{D}_{3}\right)$ and, consequently, it does not make sense to use $P_{1}^{r} v(x ; r, t)-v_{h \Delta r}(x ; r, t)$ for such type of decomposition; however, recalling the interpolant $I_{0}^{x}: H_{r}^{1,1}\left(D_{2} \times(0, R(\cdot))\right) \rightarrow$ $V_{h}^{(0)}\left(\bar{D}_{2}\right) \otimes H_{r}^{1}(0, R(\cdot))$ defined in (34) and further assuming that $P_{1}^{r} v\left(x ; r_{j}, t\right) \in$ $H^{1}\left(D_{2}\right)$, then it follows that $P_{1}^{r} v(x ; r, t) \in H_{r}^{1,1}\left(D_{2} \times(0, R(\cdot))\right)$ and, therefore, we can define $I_{0}^{x} P_{1}^{r} v(x ; r, t)$ as

$$
I_{0}^{x} P_{1}^{r} v(x ; r, t)=\sum_{l=1}^{M_{2}} \sum_{j=1}^{M^{(l)}} P_{1}^{r} v\left(x_{l} ; r_{j}, t\right) \alpha_{j}^{(l)}(r) \chi_{l}(x)
$$

this expression implies that $I_{0}^{x} P_{1}^{r} v(x ; r, t) \in V_{h \Delta r}\left(\bar{D}_{3}\right)$, so it makes sense to set

$$
\theta_{v}(x ; r, t)=I_{0}^{x} P_{1}^{r} v(x ; r, t)-v_{h \Delta r}(x ; r, t) .
$$

Now, using again the extended $P_{1}^{r}$ elliptic projector we define

$$
\rho_{v}(x ; r, t)=v(x ; r, t)-P_{1}^{r} v(x ; r, t) \in H_{r}^{1,1}\left(D_{2} \times(0, R(\cdot))\right),
$$

and consequently

$$
I_{0}^{x} \rho_{v}(x ; r, t)=I_{0}^{x} v(x ; r, t)-I_{0}^{x} P_{1}^{r} v(x ; r, t) .
$$


Then, from all these considerations we can write that

$$
v(x ; r, t)-v_{h \Delta r}(x ; r, t)=\left(v-I_{0}^{x} v\right)(x ; r, t)+I_{0}^{x} \rho_{v}(x ; r, t)+\theta_{v}(x ; r, t) .
$$

From (57) and (58) it follows that

$$
\left\|u(t)-u_{h}(t)\right\|_{L^{2}\left(D_{1}\right)} \leq\left\|\rho_{u}(t)\right\|_{L^{2}\left(D_{1}\right)}+\left\|\theta_{u}(t)\right\|_{L^{2}\left(D_{1}\right)}
$$

and

$$
\begin{aligned}
& \left\|v(t)-v_{h \Delta r}(t)\right\|_{L^{2}\left(D_{2}, L_{r}^{2}(0, R(\cdot))\right)} \leq\left\|v(t)-I_{0}^{x} v(t)\right\|_{L^{2}\left(D_{2}, L_{r}^{2}(0, R(\cdot))\right)} \\
& \quad+\left\|I_{0}^{x} \rho_{v}(t)\right\|_{L^{2}\left(D_{2}, L_{r}^{2}(0, R(\cdot))\right)}+\left\|\theta_{v}(t)\right\|_{L^{2}\left(D_{2}, L_{r}^{2}(0, R(\cdot))\right)} .
\end{aligned}
$$

The estimates for $\rho_{u}$ and $\rho_{v}$ are given in (29) and (33) respectively, i.e.,

$$
\left\{\begin{array}{l}
\left\|\rho_{u}(t)\right\|_{L^{2}\left(D_{1}\right)} \leq C h^{2}\|u(t)\|_{H^{2}\left(D_{1}\right)}, \\
\left\|\rho_{v}(t)\right\|_{L^{2}\left(D_{2}, L_{r}^{2}(0, R(\cdot))\right)} \leq C \Delta r^{2}\|v(t)\|_{L^{2}\left(D_{2}, H_{r}^{2}(0, R(\cdot))\right)},
\end{array}\right.
$$

then, it remains to calculate the estimates for $\theta_{u}$ and $\theta_{v}$; but before going into the details of such calculations, we present new estimates for $\left\|J-J_{h}\right\|_{L^{2}\left(D_{2}\right)}^{2}$ and $\left\|v_{s}(t)-v_{s h}(t)\right\|_{L^{2}\left(D_{2}\right)}^{2}$, which depend on $\theta_{u}$ and $\theta_{v}$ respectively, and will be useful for the subsequent part of the analysis.

Lemma 11 Assuming that the regularity assumptions required in the estimates hold, there exist an arbitrarily small positive number $\epsilon$ and constants $C$ and $C(\epsilon)$ independent of $h$ and $\Delta r$ such that

$$
\begin{aligned}
\left\|v_{S}(t)-v_{s h}(t)\right\|_{L^{2}\left(D_{2}\right)}^{2} \leq & C h^{2}\left|v_{S}(t)\right|_{H^{1}\left(D_{2}\right)}^{2}+C(\epsilon) \Delta r^{2}\|v(t)\|_{L^{2}\left(D_{2}, H_{r}^{2}\left(0, R_{s}(\cdot)\right)\right)}^{2} \\
& +C(\epsilon)\left\|\theta_{v}(t)\right\|_{L^{2}\left(D_{2}, L_{r}^{2}\left(0, R_{s}(\cdot)\right)\right)}^{2}+\epsilon\left\|\frac{\partial \theta_{v}(t)}{\partial r}\right\|_{L^{2}\left(D_{2}, L_{r}^{2}\left(0, R_{s}(\cdot)\right)\right)}^{2}
\end{aligned}
$$

and

$$
\begin{aligned}
\left\|J-J_{h}\right\|_{L^{2}\left(D_{2}\right)}^{2} \leq & C h^{2}\left(\left\|\phi_{1}(t)\right\|_{H^{2}\left(D_{1}\right)}^{2}+\left\|\phi_{2}(t)\right\|_{H^{2}\left(D_{2}\right)}^{2}+h^{2}\|u(t)\|_{H^{2}\left(D_{1}\right)}^{2}\right) \\
& +C\left\|v_{s}(t)-v_{s h}(t)\right\|_{L^{2}\left(D_{2}\right)}^{2}+C\left\|\theta_{u}(t)\right\|_{L^{2}\left(D_{1}\right)}^{2} .
\end{aligned}
$$

Proof To calculate the estimate (60) we set $v_{s}-v_{s h}=\left(v_{s}-I_{h}^{0} v_{s}\right)+\left(I_{h}^{0} v_{s}-v_{s h}\right)$, so using (27) it follows that

$$
\left\|v_{s}(t)-v_{s h}(t)\right\|_{L^{2}\left(D_{2}\right)}^{2}=C h^{2}\left|v_{s}(t)\right|_{H^{1}\left(D_{2}\right)}^{2}+2\left\|I_{h}^{0} v_{s}(t)-v_{s h}(t)\right\|_{L^{2}\left(D_{2}\right)}^{2} .
$$


Since $I_{h}^{0} v_{s}-v_{s h}=\left(I_{h}^{0} v-v_{h \Delta r}\right)\left(x ; R_{s}(x), t\right)=\left(I_{0}^{x} \rho_{v}+\theta_{v}\right)\left(x ; R_{S}(x), t\right)$ (recalling the definition of $\theta_{v}$ ) then by virtue of the definition of the $L^{2}$-norm for functions of $V_{h}^{0}\left(\bar{D}_{2}\right)$ presented in Sect. 3, we have that

$$
\left\|I_{h}^{0} v_{S}(t)-v_{s h}(t)\right\|_{L^{2}\left(D_{2}\right)}^{2} \leq 2 \sum_{l=1}^{M_{2}} \widehat{h}_{l}\left(\rho_{v}^{2}\left(x_{l} ; R_{S}\left(x_{l}\right), t\right)+\theta_{v}^{2}\left(x_{l} ; R_{S}\left(x_{l}\right), t\right)\right) .
$$

To estimate $\rho_{v}^{2}\left(x_{l} ; R_{s}\left(x_{l}\right), t\right)$ and $\theta_{v}^{2}\left(x_{l} ; R_{s}\left(x_{l}\right), t\right)$ we make use of Lemma 4 noting that there exists a real number $a, 0<a<R_{S}\left(x_{l}\right)$, such that $\rho_{v}^{2}\left(x_{l} ; R_{S}\left(x_{l}\right), t\right) \leq$ $\left\|\rho_{v}\left(x_{l} ; r, t\right)\right\|_{L^{\infty}\left(a, R\left(x_{l}\right)\right)}^{2}$ and $\theta_{v}^{2}\left(x_{l} ; R_{s}\left(x_{l}\right), t\right) \leq\left\|\theta_{v}\left(x_{l} ; r, t\right)\right\|_{L^{\infty}\left(a, R\left(x_{l}\right)\right)}^{2}$, thus by virtue of (37) it follows that there are a real number $\epsilon$ and positive constant $C(\epsilon)>\epsilon$ such that

$$
\begin{aligned}
\sum_{l=1}^{M_{2}} \widehat{h}_{l} \rho_{v}^{2}\left(x_{l} ; R_{S}\left(x_{l}\right), t\right) \leq & \epsilon \sum_{l=1}^{M_{2}} \widehat{h}_{l} \int_{0}^{R_{s}\left(x_{l}\right)} r^{2}\left|\frac{\partial \rho_{v}\left(x_{l} ; r, t\right)}{\partial r}\right|^{2} d r \\
& +C(\epsilon) \sum_{l=1}^{M_{2}} \widehat{h}_{l} \int_{0}^{R_{s}\left(x_{l}\right)} r^{2}\left|\rho_{v}\left(x_{l} ; r, t\right)\right|^{2} d r \\
\leq & C(\epsilon)\left\|I_{0}^{x} \rho_{v}(t)\right\|_{L^{2}\left(D_{2}, H_{r}^{1}\left(0, R_{s}(\cdot)\right)\right)}^{2} \\
\leq & C(\epsilon)\left\|\rho_{v}(t)\right\|_{L^{2}\left(D_{2}, H_{r}^{1}\left(0, R_{s}(x)\right)\right)} \\
\leq & C(\epsilon) \Delta r^{2}\|v(t)\|_{L^{2}\left(D_{2}, H_{r}^{2}\left(0, R_{s}(\cdot)\right)\right)}^{2}
\end{aligned}
$$

because by approximation theory $\left\|I_{0}^{x} \rho_{v}(t)\right\|_{L^{2}\left(D_{2}, H_{r}^{1}\left(0, R_{s}(x)\right)\right)} \leq C$ $\left\|\rho_{v}(t)\right\|_{L^{2}\left(D_{2}, H_{r}^{1}\left(0, R_{s}(x)\right)\right)}$, this latter term being estimated according to (33). Similarly,

$$
\begin{aligned}
\sum_{l=1}^{M_{2}} \widehat{h}_{l} \theta_{v}^{2}\left(x_{l} ; R_{S}\left(x_{l}\right), t\right) \leq & \epsilon \sum_{l=1}^{M_{2}} \widehat{h}_{l} \int_{0}^{R_{s}\left(x_{l}\right)} r^{2}\left|\frac{\partial \theta_{v}\left(x_{l} ; r, t\right)}{\partial r}\right|^{2} d r \\
& +C(\epsilon) \sum_{l=1}^{M_{2}} \widetilde{h}_{l} \int_{0}^{R_{s}\left(x_{l}\right)} r^{2}\left|\theta_{v}\left(x_{l} ; r, t\right)\right|^{2} d r \\
\leq & \epsilon\left|\theta_{v}(t)\right|_{L^{2}\left(D_{2}, H_{r}^{1}\left(0, R_{s}(\cdot)\right)\right)}^{2}+C(\epsilon)\left\|\theta_{v}(t)\right\|_{L^{2}\left(D_{2}, L_{r}^{2}\left(0, R_{s}(\cdot)\right)\right)}^{2}
\end{aligned}
$$

So, putting these bounds together the result (60) follows. To calculate the estimate (61) we notice that by virtue of (40) and Theorem 10

$$
\begin{aligned}
\left\|J-J_{h}\right\|_{L^{2}\left(D_{2}\right)}^{2} \leq & C\left(h^{2}\left(\left\|\phi_{1}(t)\right\|_{H^{2}\left(D_{1}\right)}^{2}+\left\|\phi_{2}(t)\right\|_{H^{2}\left(D_{2}\right)}^{2}\right)\right. \\
& \left.+\left\|u(t)-u_{h}(t)\right\|_{L^{2}\left(D_{1}\right)}^{2}+\left\|v_{s}(t)-v_{s h}(t)\right\|_{L^{2}\left(D_{2}\right)}^{2}\right) .
\end{aligned}
$$


Since $u-u_{h}=\rho_{u}+\theta_{u}$, then taking into account (29)

$$
\left\|u(t)-u_{h}(t)\right\|_{L^{2}\left(D_{1}\right)}^{2} \leq C h^{4}\|u(t)\|_{H^{2}\left(D_{1}\right)}^{2}+2\left\|\theta_{u}\right\|_{L^{2}\left(D_{1}\right)}^{2},
$$

so the result (61) follows.

Next, we calculate an estimate for $\theta_{v}$. To this end, we obtain, based on equation (11), the integral equation for $I_{0}^{x} v(x ; r, t)$ that will be used for that purpose. Thus, for each one of the mesh points $\left\{x_{l}\right\}_{l=1}^{M_{2}}$ of $D_{2 h}$ the equation (11) reads

$$
\begin{gathered}
\int_{0}^{R_{s}\left(x_{l}\right)} \frac{\partial v^{(l)}}{\partial t} w^{(l)} r^{2} d r+\int_{0}^{R_{s}\left(x_{l}\right)} k_{2} \frac{\partial v^{(l)}}{\partial r} \frac{\partial w^{(l)}}{\partial r} r^{2} d r \\
=-\left.\left(R_{s}^{2}(x) a_{2}^{-1}(x) F^{-1} J w^{(l)}\left(R_{s}(x)\right)\right)\right|_{x=x_{l}},
\end{gathered}
$$

where $v^{(l)}:=v\left(x_{l} ; r, t\right) \in H_{r}^{1}\left(0, R_{s}\left(x_{l}\right)\right.$ and $w^{(l)}:=w\left(x_{l} ; r\right) \in H_{r}^{1}\left(0, R_{S}\left(x_{l}\right)\right)$. Using the nodal basis functions $\left\{\chi_{l}(x)\right\}_{l=1}^{M_{2}}$ of the finite element space $V_{h}^{(0)}\left(\bar{D}_{2}\right)$ we can write

$$
I_{0}^{x} v(x ; r, t)=\sum_{l}^{M_{2}} v^{(l)}(r, t) \chi_{l}(x) \text { and } w(x ; r)=\sum_{l=1}^{M_{2}} w^{(l)}(r) \chi_{l}(x) .
$$

Now, noting that

$$
\int_{D_{2}} \int_{0}^{R_{S}(x)}\left(I_{0}^{x} v\right) w r^{2} d r d x=\sum_{l=1}^{M_{2}} \int_{D_{2}} \chi_{l}^{2}(x)\left(\int_{0}^{R_{S}(x)} v^{(l)}(r, t) w^{(l)}(r) r^{2} d r\right) d x
$$

and for $x \in \widehat{e}_{l}, R_{S}(x)=R_{S}\left(x_{l}\right)$, then it follows that

$$
\sum_{l=1}^{M_{2}} \int_{D_{2}} \chi_{l}^{2}(x)\left(\int_{0}^{R_{S}(x)} v^{(l)}(r, t) w^{(l)}(r) r^{2} d r\right) d x=\sum_{l=1}^{M_{2}} \widehat{h}_{l} \int_{0}^{R_{S}\left(x_{l}\right)} v^{(l)}(r, t) w^{(l)}(r) r^{2} d r .
$$

Hence, (63) becomes

$$
\begin{aligned}
& \int_{D_{2}} \int_{0}^{R_{s}(x)}\left(\frac{\partial I_{0}^{x} v}{\partial t} w+k_{2} \frac{\partial I_{0}^{x} v}{\partial r} \frac{\partial w}{\partial r}\right) r^{2} d r d x \\
& =-\int_{D_{2}} I_{0}^{x}\left(R_{s}^{2}(x) a_{2}^{-1}(x) F^{-1} J w\left(x ; R_{S}(x)\right)\right) d x
\end{aligned}
$$

We proceed to formulate the equation for $\theta_{v}$. From (58) it follows that $v_{h \Delta r}=I_{0}^{x} v-$ $\left(I_{0}^{x} \rho_{v}+\theta_{v}\right)$, then replacing this expression for $v_{h \Delta r}$ in (16) and using (32) and (64) it follows that for all $w_{h \Delta r} \in V_{h \Delta r}\left(\bar{D}_{3}\right)$, 


$$
\begin{aligned}
& \int_{D_{2}} \int_{0}^{R_{s}(x)}\left(\frac{\partial \theta_{v}}{\partial t} w_{h \Delta r}+k_{2} \frac{\partial \theta_{v}}{\partial r} \frac{\partial w_{h \Delta r}}{\partial r}\right) r^{2} d r d x \\
& =\lambda \int_{D_{2}} \int_{0}^{R_{s}(x)} I_{0}^{x} \rho_{v} w_{h \Delta r} r^{2} d r d x-\int_{D_{2}} \int_{0}^{R_{s}(x)} \frac{\partial I_{0}^{x} \rho_{v}}{\partial t} w_{h \Delta r} r^{2} d r d x \\
& \quad-\int_{D_{2}} I_{h}^{0}\left(R_{s}^{2}(x) a_{2}^{-1}(x) F^{-1}\left(J(x)-J_{h}(x)\right) w_{s h}(x)\right) d x \\
& \left.\quad+\int_{D_{2}}\left(R_{s}^{2}(x) a_{2}^{-1}(x) F^{-1} J_{h}(x)-I_{h}^{0}\left(R_{s}^{2}(x) a_{2}^{-1} F^{-1}(x) J_{h}(x)\right)\right) w_{s h}(x) d x 65\right)
\end{aligned}
$$

where we have made use of the following properties of the interpolant $I_{0}^{x}$ : (i) for $w_{h \Delta r}(x, r) \in V_{h \Delta r}\left(\bar{D}_{3}\right), w_{h \Delta r}(x, r)=I_{0}^{x} w_{h \Delta r}(x, r)$, and (ii) when $r=R_{s}(x)$ , we can define the function $w_{s h}(x)=w_{h \Delta r}\left(x, R_{s}(x)\right)$ such that $w_{s h}(x)=$ $I_{0}^{x} w_{h \Delta r}\left(x, R_{S}(x)\right)=I_{h}^{0} w_{s h}(x)$. Setting $w_{h \Delta r}=\theta_{v}$ yields

$$
\begin{aligned}
& \frac{1}{2} \frac{d}{d t}\left\|\theta_{v}(t)\right\|_{L^{2}\left(D_{2}, L_{r}^{2}\left(0, R_{s}(\cdot)\right)\right)}^{2}+\underline{k_{2}}\left\|\frac{\partial \theta_{v}(t)}{\partial r}\right\|_{L^{2}\left(D_{2}, L_{r}^{2}\left(0, R_{s}(\cdot)\right)\right)}^{2} \\
& \quad \leq \lambda\left\|I_{0}^{x} \rho_{v}(t)\right\|_{L^{2}\left(D_{2}, L_{r}^{2}\left(0, R_{s}(\cdot)\right)\right)}\left\|\theta_{v}(t)\right\|_{L^{2}\left(D_{2}, L_{r}^{2}\left(0, R_{s}(\cdot)\right)\right)} \\
& \quad+\left\|\frac{\partial I_{0}^{x} \rho_{v}(t)}{\partial t}\right\|_{L^{2}\left(D_{2}, L_{r}^{2}\left(0, R_{s}(\cdot)\right)\right)}\left\|\theta_{v}(t)\right\|_{L^{2}\left(D_{2}, L_{r}^{2}\left(0, R_{s}(\cdot)\right)\right)} \\
& \quad+C\left\|I_{h}^{(0)}\left(\left(J-J_{h}\right) \theta_{v s}(t)\right)\right\|_{L^{1}\left(D_{2}\right)}+C\left\|\left(J_{h}-I_{(0)}^{0} J_{h}\right) \theta_{v s}(t)\right\|_{L^{1}\left(D_{2}\right)} \equiv \sum_{i=1}^{4} R_{i},
\end{aligned}
$$

where, $\theta_{v s}(t)=\theta_{v}\left(x ; R_{S}(x), t\right)$ is the value of $\theta_{v}$ on the surface of the sphere of radius $R_{x}(x)$ associated with the point $\{x\}$ of $D_{2}$.

Lemma 12 There exists a constant $C$ independent of $h$ and $\Delta r$, but depending on $k_{1}$ and $\underline{k_{2}}$, such that

$$
\begin{aligned}
\frac{d}{d t} & \left\|\theta_{v}(t)\right\|_{L^{2}\left(D_{2}, L_{r}^{2}\left(0, R_{s}(\cdot)\right)\right)}^{2}+\underline{k_{2}}\left\|\frac{\partial \theta_{v}(t)}{\partial r}\right\|_{L^{2}\left(D_{2}, L_{r}^{2}\left(0, R_{s}(\cdot)\right)\right)}^{2} \\
\leq & C h^{2}\left(\left\|\phi_{1}(t)\right\|_{H^{2}\left(D_{1}\right)}^{2}+\left\|\phi_{2}(t)\right\|_{H^{2}\left(D_{2}\right)}^{2}\right) \\
& +C h^{2}\left(\left|v_{s}(t)\right|_{H^{1}\left(D_{2}\right)}^{2}+h^{2}\|u(t)\|_{H^{2}\left(D_{1}\right)}^{2}+\left\|\frac{\partial J}{\partial x}\right\|_{L^{2}\left(D_{2}\right)}^{2}\right) \\
& +C \Delta r^{2}\left(\|v(t)\|_{L^{2}\left(D_{2}, H_{r}^{2}\left(0, R_{s}(\cdot)\right)\right)}^{2}+\Delta r^{2}\left\|\frac{\partial v(t)}{\partial t}\right\|_{L^{2}\left(D_{2}, H_{r}^{2}\left(0, R_{s}(\cdot)\right)\right)}^{2}\right) \\
& +C\left(\left\|\theta_{u}(t)\right\|_{L^{2}\left(D_{1}\right)}^{2}+\left\|\theta_{v}(t)\right\|_{L^{2}\left(D_{2}, L_{r}^{2}\left(0, R_{s}(\cdot)\right)\right)}\right) .
\end{aligned}
$$


Proof We bound the terms $R_{1}, \ldots, R_{4}$ on the right hand side of (66). Noting that $\left\|I_{0}^{x} \rho_{v}(t)\right\|_{L^{2}\left(D_{2}, L_{r}^{2}\left(0, R_{S}(\cdot)\right)\right)} \leq C\left\|\rho_{v}(t)\right\|_{L^{2}\left(D_{2}, L_{r}^{2}\left(0, R_{S}(\cdot)\right)\right)}$, then by virtue of Young inequality and (33) it follows that

$$
R_{1} \leq C \Delta r^{4}\|v(t)\|_{L^{2}\left(D_{2}, H_{r}^{2}\left(0, R_{s}(\cdot)\right)\right)}^{2}+C\left\|\theta_{v}(t)\right\|_{L^{2}\left(D_{2}, L_{r}^{2}\left(0, R_{S}(\cdot)\right)\right)}^{2} .
$$

To bound $R_{2}$ we notice that

$$
\begin{aligned}
\left\|\frac{\partial I_{0}^{x} \rho_{v}(t)}{\partial t}\right\|_{L^{2}\left(D_{2}, L_{r}^{2}\left(0, R_{s}(\cdot)\right)\right)} \leq C & \left\|\frac{\partial \rho_{v}(t)}{\partial t}\right\|_{L^{2}\left(D_{2}, L_{r}^{2}\left(0, R_{s}(\cdot)\right)\right)} \\
& =C\left\|\left(I-P_{1}^{r}\right) \frac{\partial v(t)}{\partial t}\right\|_{L^{2}\left(D_{2}, L_{r}^{2}\left(0, R_{s}(\cdot)\right)\right)} \\
& \leq C \Delta r^{2}\left\|\frac{\partial v(t)}{\partial t}\right\|_{L^{2}\left(D_{2}, H_{r}^{2}\left(0, R_{s}(\cdot)\right)\right)} .
\end{aligned}
$$

Hence, by Young inequality it follows that

$$
R_{2} \leq C \Delta r^{4}\left\|\frac{\partial v(t)}{\partial t}\right\|_{L^{2}\left(D_{2}, H_{r}^{2}\left(0, R_{s}(\cdot)\right)\right)}^{2}+C\left\|\theta_{v}(t)\right\|_{L^{2}\left(D_{2}, L_{r}^{2}\left(0, R_{S}(\cdot)\right)\right)}^{2} .
$$

We bound the term $R_{3}$. Thus, we have that

$$
\begin{aligned}
C\left\|I_{h}^{0}\left(\left(J-J_{h}\right) \theta_{v s}(t)\right)\right\|_{L^{1}\left(D_{2}\right)} & =C \sum_{l=1}^{M_{2}} \widehat{h}_{l}\left|\left(J\left(x_{l}\right)-J_{h}\left(x_{l}\right)\right) \theta_{v}\left(x_{l} ; R_{S}\left(x_{l}\right), t\right)\right| \\
& \leq \frac{C^{2}}{2} \sum_{l=1}^{M_{2}} \widehat{h}_{l}\left(J\left(x_{l}\right)-J_{h}\left(x_{l}\right)\right)^{2}+\frac{1}{2} \sum_{l=1}^{M_{2}} \widehat{h}_{l} \theta_{v}^{2}\left(x_{l} ; R_{S}\left(x_{l}\right), t\right) \\
& \leq \frac{C^{2}}{2}\left\|I_{h}^{0}\left(J-J_{h}\right)\right\|_{L^{2}\left(D_{2}\right)}^{2}+\frac{1}{2} \sum_{l=1}^{M_{2}} \widehat{h}_{l} \theta_{v}^{2}\left(x_{l} ; R_{S}\left(x_{l}\right), t\right) .
\end{aligned}
$$

Estimating the last term on the right hand side of this inequality as we did before in the proof of Lemma 11, see (62), and noting that $\left\|I_{h}^{0}\left(J-J_{h}\right)\right\|_{L^{2}\left(D_{2}\right)} \leq$ $C\left\|J-J_{h}\right\|_{L^{2}\left(D_{2}\right)}^{2}$, it readily follows that

$$
R_{3} \leq C\left\|J-J_{h}\right\|_{L^{2}\left(D_{2}\right)}^{2}+C(\epsilon)\left\|\theta_{v}(t)\right\|_{L^{2}\left(D_{2}, L_{r}^{2}\left(0, R_{s}(\cdot)\right)\right)}^{2}+\epsilon\left\|\frac{\partial \theta_{v}(t)}{\partial r}\right\|_{L^{2}\left(D_{2}, L_{r}^{2}\left(0, R_{s}(\cdot)\right)\right)}^{2} .
$$

To bound $R_{4}$ we notice that $J_{h}-I_{h}^{(0)} J_{h}=\left(J_{h}-J\right)+\left(J-I_{h}^{(0)} J\right)+I_{h}^{(0)}\left(J-J_{h}\right)$, so by the triangle inequality it follows that

$$
C\left\|\left(J_{h}-I_{h}^{(0)} J_{h}\right) \theta_{v s}(t)\right\|_{L^{1}\left(D_{2}\right)} \leq C\left\|\left(J_{h}-J\right) \theta_{v s}(t)\right\|_{L^{1}\left(D_{2}\right)}
$$




$$
+C\left\|\left(J-I_{h}^{(0)} J\right) \theta_{v s}(t)\right\|_{L^{1}\left(D_{2}\right)}+C\left\|I_{h}^{(0)}\left(J-J_{h}\right) \theta_{v s}(t)\right\|_{L^{1}\left(D_{2}\right)} .
$$

Noting that $\|a b\|_{L^{1}\left(D_{2}\right)} \leq \frac{\varepsilon}{2}\|a\|_{L^{2}\left(D_{2}\right)}^{2}+\frac{1}{2 \varepsilon}\|b\|_{L^{2}\left(D_{2}\right)}^{2}$ and applying the same argument as we have just done to bound $R_{3}$, we obtain that

$$
\begin{aligned}
\left\|\left(J_{h}-I_{h}^{0} J_{h}\right) \theta_{v s}(t)\right\|_{L^{1}\left(D_{2}\right)} \leq & C\left(\left\|J-J_{h}\right\|_{L^{2}\left(D_{2}\right)}^{2}+\left\|J-I_{h}^{0} J\right\|_{L^{2}\left(D_{2}\right)}^{2}\right) \\
& +\sum_{l=1}^{M_{2}} \widehat{h}_{l} \theta_{v}^{2}\left(x_{l} ; R_{S}\left(x_{l}\right), t\right) .
\end{aligned}
$$

We bound the last term of this inequality as we have done for $R_{3}$, and by virtue of assumption A3 set $\left\|J-I_{h}^{(0)} J\right\|_{L^{2}\left(D_{2}\right)}^{2} \leq C h^{2}\left\|\frac{\partial J}{\partial x}\right\|_{L^{2}\left(D_{2}\right)}^{2}$. Hence,

$$
\begin{aligned}
R_{4} \leq & C(\epsilon)\left\|\theta_{v}(t)\right\|_{L^{2}\left(D_{2}, L_{r}^{2}\left(0, R_{s}(\cdot)\right)\right)}^{2}+\epsilon\left|\frac{\partial \theta_{v}(t)}{\partial r}\right|_{L^{2}\left(D_{2}, L_{r}^{2}\left(0, R_{s}(\cdot)\right)\right)}^{2} \\
& +C\left\|J-J_{h}\right\|_{L^{2}\left(D_{2}\right)}^{2}+C h^{2}\left\|\frac{\partial J}{\partial x}\right\|_{L^{2}\left(D_{2}\right)}^{2} \cdot
\end{aligned}
$$

Letting $\epsilon=k_{2} / 8$ in (61), (70) and (71), and replacing (68)-(71) in (66), the result (67) follows.

Next, we proceed to calculate an estimate for $\theta_{u}$. Thus, subtracting (15) from (10) it readily follows that

$$
\begin{aligned}
\int_{D_{1}} \frac{\partial \theta_{u}}{\partial t} w_{h} d x+\int_{D_{1}} k_{1} \frac{\partial \theta_{u}}{\partial x} \frac{d w_{h}}{d x} d x= & \lambda \int_{D_{1}} \rho_{u} w_{h} d x-\int_{D_{1}} \frac{\partial \rho_{u}}{\partial t} w_{h} d x \\
& +\int_{D_{1}} a_{1}\left(J-J_{h}\right) w_{h} d x
\end{aligned}
$$

Setting $w_{h}=\theta_{u}$ in this equation yields

$$
\begin{aligned}
\frac{1}{2} \frac{d}{d t}\left\|\theta_{u}(t)\right\|_{L^{2}\left(D_{1}\right)}^{2}+\underline{k_{1}}\left\|\frac{\partial \theta_{u}(t)}{\partial x}\right\|_{L^{2}\left(D_{1}\right)}^{2} \leq & C\left(\left\|\rho_{u}(t)\right\|_{L^{2}\left(D_{1}\right)}^{2}+\left\|\frac{\partial \rho_{u}(t)}{\partial t}\right\|_{L^{2}\left(D_{1}\right)}^{2}\right) \\
& +C\left\|J-J_{h}\right\|_{L^{2}\left(D_{1}\right)}^{2}+C\left\|\theta_{u}(t)\right\|_{L^{2}\left(D_{1}\right)}^{2}
\end{aligned}
$$

By virtue of Lemma 11 we have the following result.

Lemma 13 There exists a constant $C$ independent of $h$ and $\Delta r$, but depending on $k_{1}$ and $\underline{k_{2}}$, such that 


$$
\begin{aligned}
\frac{d}{d t} & \left\|\theta_{u}(t)\right\|_{L^{2}\left(D_{1}\right)}^{2}+\underline{k_{1}}\left\|\frac{\partial \theta_{u}(t)}{\partial x}\right\|_{L^{2}\left(D_{1}\right)}^{2} \\
\leq & C\left\{h^{2}\left(\left\|\phi_{1}(t)\right\|_{H^{2}\left(D_{1}\right)}^{2}+\left\|\phi_{2}(t)\right\|_{H^{2}\left(D_{2}\right)}^{2}+\left|v_{s}(t)\right|_{H^{1}\left(D_{2}\right)}^{2}\right)\right. \\
& \left.+h^{4}\left(\|u(t)\|_{H^{2}\left(D_{1}\right)}^{2}+\left\|\frac{\partial u(t)}{\partial t}\right\|_{H^{2}\left(D_{1}\right)}^{2}\right)+\Delta r^{2}\|v(t)\|_{L^{2}\left(D_{2}, H_{r}^{2}\left(0, R_{s}(\cdot)\right)\right)}^{2}\right\} \\
& +C\left(\left\|\theta_{u}(t)\right\|_{L^{2}\left(D_{1}\right)}^{2}+\left\|\theta_{v}(t)\right\|_{L^{2}\left(D_{2}, L_{r}^{2}\left(0, R_{s}(\cdot)\right)\right)}\right)+\frac{k_{2}}{\frac{4}{4}}\left\|\frac{\partial \theta_{v}(t)}{\partial r}\right\|_{L^{2}\left(D_{2}, L_{r}^{2}\left(0, R_{s}(\cdot)\right)\right)}
\end{aligned} .
$$

We are now in a position to establish the main result of this subsection.

Theorem 14 Let $\left(u, v, \phi_{1}, \phi_{2}\right)$ and $\left(u_{h}, v_{h \Delta r}, \phi_{1 h}, \phi_{2 h}\right)$ be the solutions to (10)-(13) and (15)-(18) respectively, with

$$
\left\|u(0)-u_{h}(0)\right\|_{L^{2}\left(D_{1}\right)} \leq C h^{2} \text { and }\left\|v(0)-v_{h \Delta r}(0)\right\|_{L^{2}\left(D_{2}, L_{r}^{2}\left(0, R_{s}(\cdot)\right)\right)} \leq C\left(h+\Delta r^{2}\right) .
$$

Furthermore, for $0 \leq t \leq T_{\text {end }}$, the following regularity assumptions hold:

$R 1) u$ and $\frac{\partial u}{\partial t} \in L^{2}\left(0, T_{\mathrm{end}} ; H^{2}\left(D_{1}\right)\right)$,

$R 2) v$ and $\frac{\partial v(t)}{\partial t} \in L^{2}\left(0, T_{\mathrm{end}} ; L^{2}\left(D_{2} ; H_{r}^{2}\left(0, R_{s}(\cdot)\right)\right)\right)$, and $v_{s} \in L^{2}\left(0, T_{\mathrm{end}} ; H^{1}\right.$ $\left.\left(D_{2}\right)\right)$,

$R 3) \phi_{1} \in L^{2}\left(0, T_{\mathrm{end}} ; H^{2}\left(D_{1}\right)\right), \phi_{2} \in L^{2}\left(0, T_{\mathrm{end}} ; H^{2}\left(D_{2}\right)\right)$, and $J \in L^{2}\left(0, T_{\mathrm{end}}\right.$; $\left.H^{1}\left(D_{2}\right)\right)$;

then there is a constant $C\left(t, \underline{k_{1}}, \underline{k_{2}}\right)$ such that

$$
\begin{gathered}
\left\|u(t)-u_{h}(t)\right\|_{L^{2}\left(D_{1}\right)}^{2}+\left\|v(t)-v_{h \Delta r}(t)\right\|_{L^{2}\left(D_{2}, L_{r}^{2}\left(0, R_{s}(\cdot)\right)\right)}^{2} \\
+\int_{0}^{t}\left\|\Phi(\tau)-\Phi_{h}(\tau)\right\|_{V}^{2} d \tau \leq C\left(h^{2}+\Delta r^{2}\right)
\end{gathered}
$$

Proof Bounding $\left\|u(t)-u_{h}(t)\right\|_{L^{2}\left(D_{1}\right)}^{2}$ as $2\left(\left\|\rho_{u}(t)\right\|^{2}+\left\|\theta_{u}(t)\right\|^{2}\right)$ and using the estimate of Lemma 11 for $\left\|v_{s}(t)-v_{s h}(t)\right\|_{L^{2}\left(D_{2}\right)}$, Theorem 10 yields

$$
\begin{aligned}
\left\|\Phi(t)-\Phi_{h}(t)\right\|_{V}^{2} \leq & C h^{2}\left|v_{S}(t)\right|_{H^{1}\left(D_{2}\right)}^{2}+C h^{4}\|u(t)\|_{H^{2}\left(D_{1}\right)}^{2}+C\left\|\theta_{u}\right\|_{L^{2}\left(D_{1}\right)}^{2} \\
& +C(\epsilon)\left\|\theta_{v}(t)\right\|_{L^{2}\left(D_{2}, L_{r}^{2}\left(0, R_{S}(\cdot)\right)\right)}^{2}+\epsilon\left\|\frac{\partial \theta_{v}(t)}{\partial r}\right\|_{L^{2}\left(D_{2}, L_{r}^{2}\left(0, R_{s}(\cdot)\right)\right)}^{2} .
\end{aligned}
$$

Considering this estimate together with those of Lemmas 12 and 13 it readily follows that 


$$
\begin{aligned}
& \frac{d}{d t}\left(\left\|\theta_{u}(t)\right\|_{L^{2}\left(D_{1}\right)}^{2}+\left\|\theta_{v}(t)\right\|_{L^{2}\left(D_{2}, L_{r}^{2}\left(0, R_{s}(\cdot)\right)\right)}^{2}\right)+\left\|\Phi(t)-\Phi_{h}(t)\right\|_{V}^{2} \leq C\left(h^{2}+\Delta r^{2}\right) \\
& \quad+C\left(\left\|\theta_{u}(t)\right\|_{L^{2}\left(D_{1}\right)}^{2}+\left\|\theta_{v}(t)\right\|_{L^{2}\left(D_{2}, L_{r}^{2}\left(0, R_{s}(\cdot)\right)\right)}^{2}\right) .
\end{aligned}
$$

Then, Gronwall inequality yields

$$
\begin{aligned}
& \left\|\theta_{u}(t)\right\|_{L^{2}\left(D_{1}\right)}^{2}+\left\|\theta_{v}(t)\right\|_{L^{2}\left(D_{2}, L_{r}^{2}\left(0, R_{s}(\cdot)\right)\right)}^{2} \\
& \quad+\int_{0}^{t}\left\|\Phi(\tau)-\Phi_{h}(\tau)\right\|_{V}^{2} d \tau \leq C\left(t, \underline{k_{1}}, \underline{k_{2}}\right)\left(h^{2}+\Delta r^{2}\right) .
\end{aligned}
$$

The terms $\left\|\theta_{u}(0)\right\|_{L^{2}\left(D_{1}\right)}^{2}$ and $\left\|\theta_{v}(0)\right\|_{L^{2}\left(D_{2}, L_{r}^{2}\left(0, R_{s}(\cdot)\right)\right)}^{2}$ are considered to be zero. From (57), (58), (59) and (35) it follows that

$$
\begin{aligned}
\| u(t)- & u_{h}(t)\left\|_{L^{2}\left(D_{1}\right)}^{2}+\right\| v(t)-v_{h \Delta r}(t) \|_{L^{2}\left(D_{2}, L_{r}^{2}\left(0, R_{S}(\cdot)\right)\right)}^{2} \\
\leq & 2\left(\left\|\rho_{u}(t)\right\|_{L^{2}\left(D_{1}\right)}+\left\|\theta_{u}(t)\right\|_{L^{2}\left(D_{1}\right)}\right) \\
& +C\left(h^{2}\|v(t)\|_{H^{1}\left(D_{2} ; L_{r}^{2}\left(0, R_{s}(\cdot)\right)\right)}+\left\|\rho_{v}(t)\right\|_{L^{2}\left(D_{2}, L_{r}^{2}\left(0, R_{S}(\cdot)\right)\right.}^{2}\right. \\
& \left.+\left\|\theta_{v}(t)\right\|_{L^{2}\left(D_{2}, L_{r}^{2}\left(0, R_{S}(\cdot)\right)\right.}^{2}\right)
\end{aligned}
$$

So, by combining this inequality with (74) we obtain (73).

\section{Fully discrete model}

We now consider the fully discrete model based on the time stepping backward Euler scheme. This scheme has been used to discretize in time the equations of the P2D model either with finite differences [12], finite volumes [11,17], or finite elements. For convenience, hereafter we shall use the notation $a^{n}:=a\left(x, t_{n}\right)$, where $n$ is a nonnegative integer and $t_{n}=n \Delta t, \Delta t$ being a uniform time step. The formulation of the fully discrete model is as follows. Assuming that at time $t_{n-1}, n=1,2, \ldots, N$, the solution $\left(u_{h}^{n-1}, v_{h \Delta r}^{n-1}, \phi_{1 h}^{n-1}, \phi_{1 h}^{n-1}\right) \in V_{h}^{(1)}\left(\bar{D}_{1}\right) \times V_{h \Delta r}\left(\bar{D}_{3}\right) \times W_{h}\left(\bar{D}_{1}\right) \times V_{h}^{(1)}\left(\bar{D}_{2}\right)$ is known, calculate $\left(u_{h}^{n}, v_{h \Delta r}^{n}, \phi_{1 h}^{n}, \phi_{1 h}^{n}\right) \in V_{h}^{(1)}\left(\bar{D}_{1}\right) \times V_{h \Delta r}\left(\bar{D}_{3}\right) \times W_{h}\left(\bar{D}_{1}\right) \times V_{h}^{(1)}\left(\bar{D}_{2}\right)$ as solution of the system

$$
\begin{aligned}
& \int_{D_{1}} \widetilde{\partial}_{t} u^{n}{ }_{h} w_{h} d x+\int_{D_{1}} k_{1} \frac{d u_{h}^{n}}{d x} \frac{d w_{h}}{d x} d x=\int_{D_{1}} a_{1} J_{h}^{n} w_{h} d x \forall w_{h} \in V_{h}^{(1)}\left(\bar{D}_{1}\right) . \\
& \left\{\begin{array}{l}
\int_{D_{2}} \int_{0}^{R_{s}(x)} \widetilde{\partial}_{t} v_{h \Delta r}^{n} w_{h \Delta r} r^{2} d r d x+\int_{D_{2}} \int_{0}^{R_{s}(x)} k_{2} \frac{\partial v^{n} h \Delta r}{\partial r} \frac{\partial w_{h \Delta r}}{\partial r} r^{2} d r d x \\
=-\int_{D_{2}} \frac{R_{s}^{2}(x) J_{h}^{n} w_{h \Delta r}\left(x, R_{s}(x)\right)}{a_{2}(x) F} d x \quad \forall w_{h \Delta r} \in V_{h \Delta r}\left(\bar{D}_{3}\right) .
\end{array}\right.
\end{aligned}
$$




$$
\begin{aligned}
& \int_{D_{1}} \kappa\left(u_{h}^{n}\right) \frac{d \phi_{1 h}^{n}}{d x} \frac{d w_{h}}{d x} d x=\int_{D_{1}} J_{h}^{n} w_{h} d x \forall w_{h} \in V_{h}^{(1)}\left(\bar{D}_{1}\right) . \\
& \int_{D_{2}} \sigma \frac{d \phi_{2 h}^{n}}{d x} \frac{d w_{h}}{d x} d x=-\int_{D_{2}}\left(J_{h}^{n}+g\right) w_{h} d x \quad \forall w_{h} \in V_{h}^{(1)}\left(\bar{D}_{2}\right) . \\
& \int_{D_{2}} J_{h}^{n} d x=0 \text { with } \int_{D_{\mathrm{a}}} J_{h}^{n} d x=I\left(t_{n}\right)=-\int_{D_{\mathrm{c}}} J_{h}^{n} d x
\end{aligned}
$$

where

$$
\begin{aligned}
\widetilde{\partial}_{t} u_{h}^{n} & =\frac{u_{h}^{n}-u_{h}^{n-1}}{\Delta t}, \quad \widetilde{\partial}_{t} v_{h \Delta r}^{n}=\frac{v_{h \Delta r}^{n}-v_{h \Delta r}^{n-1}}{\Delta t} \\
J_{h}^{n} & =J\left(x, u_{h}^{n}, v_{s h}^{n}, \eta_{h}^{n}\right)=a_{2}(x) i_{0 h}^{n} \sinh \left(\beta \eta_{h}^{n}\right), i_{0 h}^{n}=i_{0}\left(u_{h}^{n}, v_{s h}^{n}\right), \\
\eta_{h}^{n} & =\phi_{1 h}^{n}-\phi_{2 h}^{n}-\alpha_{h}^{n} \ln u_{h}^{n}-\bar{U}_{h}\left(v_{s h}^{n}\right), \alpha_{h}^{n}=\alpha\left(u_{h}^{n}\right) .
\end{aligned}
$$

\subsection{On the existence and uniqueness of the solution of the fully discrete model}

To prove that the system (75)-(78) has a unique solution, we first show that assuming $\left(u_{h}^{n}, v_{h \Delta r}^{n}, v_{s h}^{n}\right) \in V_{h}^{(1)}\left(\bar{D}_{1}\right) \times V_{h \Delta r}\left(\bar{D}_{3}\right) \times V_{h}^{(0)}\left(\bar{D}_{2}\right)$ and the assumptions A1-A4 hold, the system (77)-(78) has a unique solution $\left(\phi_{1 h}^{n}, \phi_{2 h}^{n}\right) \in W_{h}\left(\bar{D}_{1}\right) \times V_{h}^{(1)}\left(\bar{D}_{2}\right)$; then, returning to the system (75)-(76) and applying a well-known consequence of Brower's fixed point theorem, which is presented as Corollary 1.1 in [8], we prove that there exists $\left(u_{h}^{n}, v_{h \Delta r}^{n}\right) \in V_{h}^{(1)}\left(\bar{D}_{1}\right) \times V_{h \Delta r}\left(\bar{D}_{3}\right)$.

Lemma 15 Assuming that for all $n,\left(u_{h}^{n}, v_{h \Delta r}^{n}, v_{s h}^{n}\right) \in V_{h}^{(1)}\left(\bar{D}_{1}\right) \times V_{h \Delta r}\left(\bar{D}_{3}\right) \times$ $V_{h}^{(0)}\left(\bar{D}_{2}\right)$, and the assumptions A1-A4 hold, then the system (77)-(78) has a unique solution $\left(\phi_{1 h}^{n}, \phi_{2 h}^{n}\right) \in W_{h}\left(\bar{D}_{1}\right) \times V_{h}^{(1)}\left(\bar{D}_{2}\right)$.

Proof Looking at (77)-(78) and in order to apply Minty-Browder theorem to prove the existence of a solution, we define the functions

$$
\widehat{\eta}_{h}^{n}:=-\alpha_{h}^{n} \ln u_{h}^{n}-\bar{U}_{h}\left(v_{s h}^{n}\right) \text { and } \widehat{J}_{h}^{n}:=J_{h}^{n}\left(x, u_{h}^{n}, v_{s h}^{n}, \widehat{\eta}_{h}^{n}\right),
$$

it is worth noticing that $\widehat{\eta}_{h}^{n}$ is equal to $\eta_{h}^{n}$ when the potentials $\phi_{1 h}^{n}$ and $\phi_{1 h}^{n}$ are zero. Now, going back to Sect. 4.2 and using $\widehat{J}_{h}^{n}$, we define the operators $\widehat{B}_{h}: V_{h} \rightarrow V_{h}^{*}$ and $A_{h}: V_{h} \rightarrow V_{h}^{*}$ as follows: for all $n=1,2, . ., N$,

$$
\left\langle\widehat{B}_{h}\left(\Phi_{h}^{n}\right), \Psi_{h}\right\rangle=\int_{D_{2}}\left(J_{h}^{n}-\widehat{J}_{h}^{n}\right)\left(\psi_{2 h}-\psi_{1 h}\right) d x \quad \forall \Psi_{h} \in V_{h} .
$$

and

$$
\left\langle A_{h}\left(\Phi_{h}^{n}\right), \Psi_{h}\right\rangle=a_{h}\left(\Phi_{h}^{n}, \Psi_{h}\right)+\left\langle\widehat{B}_{h}\left(\Phi_{h}^{n}\right), \Psi_{h}\right\rangle
$$


Notice that when $\Phi_{h}^{n}=(0,0),\left\langle\widehat{B}_{h}\left(\Phi_{h}^{n}\right), \Psi_{h}\right\rangle=0$ because $J_{h}^{n}=\widehat{J}_{h}^{n}$. Now, we can recast (77)-(78) as follows. Find $\Phi_{h}^{n}:=\left(\phi_{1}^{n}, \phi_{1}^{n}\right) \in W_{h}\left(\bar{D}_{1}\right) \times V_{h}^{(1)}\left(\bar{D}_{2}\right)$ such that

$$
\left\langle A_{h}\left(\Phi_{h}^{n}\right), \Psi_{h}\right\rangle=-\int_{D_{2}} g \psi_{2 h} d x-\int_{D_{2}} \widehat{J}_{h}^{n}\left(\psi_{2 h}-\psi_{1 h}\right) d x \quad \forall \Psi_{h} \in V_{h}
$$

We can prove, using the same arguments as in Lemma 8 , that the operator $\widehat{B}_{h}$ is monotone, bounded and continuous satisfying an inequality as (46); since the bilinear form $a_{h}$ is continuous and semi-definite positive, then it follows that the operator $A_{h}$ is monotone, bounded and continuous satisfying an inequality as (46). In order to prove that (81) has a solution it remains to show that $A_{h}$ is coercive, i.e., $\forall \Phi_{h}^{n} \in$ $W_{h}\left(\bar{D}_{1}\right) \times V_{h}^{(1)}\left(\bar{D}_{2}\right)$, there exists a positive constant $\alpha$ such that

$$
\left\langle A_{h}\left(\Phi_{h}^{n}\right), \Phi_{h}^{n}\right\rangle \geq \alpha\left\|\Phi_{h}^{n}\right\|_{V}^{2}
$$

This can be easily done by considering the following facts: 1) $A_{h}$ is monotone; 2) it is easy to check, using the same arguments as in Theorem 10 to prove (51), that $\forall \Phi_{h}^{n}$, $\bar{\Phi}_{h}^{n} \in W_{h}\left(\bar{D}_{1}\right) \times V_{h}^{(1)}\left(\bar{D}_{2}\right)$

$$
a_{h}\left(\Phi_{h}^{n}-\bar{\Phi}_{h}^{n}, \Phi_{h}^{n}-\bar{\Phi}_{h}^{n}\right)+\left\langle\widehat{B}_{h}\left(\Phi_{h}^{n}\right)-\widehat{B}_{h}\left(\Phi_{h}^{n}\right), \Phi_{h}^{n}-\bar{\Phi}_{h}^{n}\right\rangle \geq \alpha\left\|\Phi_{h}^{n}-\bar{\Phi}_{h}^{n}\right\|_{V}^{2}
$$

then taking $\bar{\Phi}_{h}^{n}=(0,0)$ it follows the coerciveness of $A_{h}$. Hence, the Minty-Browder theorem [18] guaranties the existence of a solution $\Phi_{h}^{n}$ of (81). To prove the uniqueness of this solution we follow the argument put forward in [19] to prove the uniqueness of the exact solution, and assume that there two solutions $\Phi_{h}^{n}:=\left(\phi_{1 h}^{n}, \phi_{2 h}^{n}\right)$ and $\bar{\Phi}_{h}^{n}:=\left(\bar{\phi}_{1 h}^{n}, \bar{\phi}_{2 h}^{n}\right)$ of (77)-(78), then setting, $z_{1 h}=\phi_{1 h}^{n}-\bar{\phi}_{1 h}^{n}$ and $z_{2 h}=\phi_{2 h}^{n}-\bar{\phi}_{2 h}^{n}$, from (77) it follows that

$$
\int_{D_{1}} \kappa\left(u_{h}^{n}\right) \frac{d z_{1 h}}{d x} \frac{d w_{h}}{d x} d x=\int_{D_{2}}\left(J_{h}^{n}-\bar{J}_{h}^{n}\right) w_{h} d x \quad \forall w_{h} \in V_{h}^{(1)}\left(\bar{D}_{1}\right)
$$

and from (78)

$$
\int_{D_{1}} \sigma \frac{d z_{2 h}}{d x} \frac{d v_{h}}{d x} d x=-\int_{D_{2}}\left(J_{h}^{n}-\bar{J}_{h}^{n}\right) v_{h} d x \forall v_{h} \in V_{h}^{(1)}\left(\bar{D}_{2}\right),
$$

where $J_{h}^{n}=J_{h}^{n}\left(x, u_{h}^{n}, v_{s h}^{n}, \eta_{h}^{n}\right)$ and $\bar{J}_{h}^{n}=J_{h}^{n}\left(x, u_{h}^{n}, v_{s h}^{n}, \bar{\eta}_{h}^{n}\right)$, with $\bar{\eta}_{h}^{n}=\bar{\phi}_{2 h}^{n}-\bar{\phi}_{1 h}^{n}-$ $\alpha_{h}^{n} \ln u_{h}^{n}-\bar{U}_{h}\left(v_{s h}^{n}\right)$. Setting $w_{h}=z_{1 h}$ and $v_{h}=z_{2 h}$ and applying the mean value theorem one readily obtains that

$$
\int_{D_{1}} \kappa\left(u_{h}^{n}\right)\left(\frac{d z_{1 h}}{d x}\right)^{2} d x+\int_{D_{1}} \sigma\left(\frac{d z_{2 h}}{d x}\right)^{2} d x+\int_{D_{2}} \frac{\partial J_{h}^{n}(\xi)}{\partial \eta^{n}}\left(z_{2 h}-z_{1 h}\right)^{2}=0,
$$


here $\frac{\partial J_{h}^{n}(\xi)}{\partial \eta^{n}}>0$ according to assumption A3. The first term of this expression implies that for all $n$,

$$
z_{1 h}=\phi_{1 h}^{n}-\bar{\phi}_{1 h}^{n}=K_{1}
$$

but the constant $K_{1}=0$ because $\phi_{1 h}^{n}$ and $\bar{\phi}_{1 h}^{n}$ are in $W_{h}\left(\bar{D}_{1}\right)$, so $\phi_{1 h}^{n}=\bar{\phi}_{1 h}^{n}$ Similarly, from the second and third terms it follows that $z_{2 h}=0$, and consequently $\phi_{2 h}^{n}=\bar{\phi}_{2 h}^{n}$. Hence, we have just proved that for all $n$ there is a unique solution $\left(\phi_{1 h}^{n}, \phi_{2 h}^{n}\right)$.

Lemma 16 Let $\left(\phi_{1 h}^{n}, \phi_{2 h}^{n}\right) \in W_{h}\left(\bar{D}_{1}\right) \times V_{h}^{(1)}\left(\bar{D}_{2}\right)$ be the solution to (77)-( 78). There exists a unique solution $\left(u_{h}^{n}, v_{h \Delta r}^{n}\right) \in V_{h}^{(1)}\left(\bar{D}_{1}\right) \times V_{h \Delta r}\left(\bar{D}_{3}\right)$ to the system (75)-(76).

Proof We start proving the existence of $u_{h}^{n} \in V_{h}^{(1)}\left(\bar{D}_{1}\right)$ as solution of (75). To this end, we write (75) as $F_{h}\left(u_{h}^{n}\right)=0$, where $F_{h}: V_{h}^{(1)}\left(\bar{D}_{1}\right) \rightarrow V_{h}^{(1)}\left(\bar{D}_{1}\right)$ is a continuous mapping defined by the relation

$$
\begin{aligned}
\int_{D_{1}} F_{h}\left(\chi_{h}\right) w_{h} d x & =\int_{D_{1}}\left(\chi_{h}-u_{h}^{n-1}\right) w_{h} d x+\Delta t \int_{D_{1}} k_{1} \frac{d \chi_{h}^{n}}{d x} \frac{d w_{h}}{d x} d x \\
& -\Delta t \int_{D_{1}} a_{1} J_{h}^{n}\left(\chi_{h}\right) w_{h} d x=0 \quad \forall w_{h} \in V_{h}^{(1)}\left(\bar{D}_{1}\right)
\end{aligned}
$$

here, $J_{h}^{n}\left(\chi_{h}\right)=J\left(x, \chi_{h}, v_{s h}, \phi_{1 h}^{n}, \phi_{2 h}^{n}, \bar{U}_{h}\left(v_{s h}\right)\right)$, with $v_{s h}$ being picked up from $S_{Q}^{*}$ because we assume that $v_{h \Delta r}^{n}$ belongs to this space; moreover, we also assume that $\chi_{h}$ is in $S_{P}$. According to Brower's fixed point theorem, the equation $F_{h}\left(\chi_{h}\right)=0$ has a solution $\chi_{h} \in B_{q}:=\left\{v_{h} \in V_{h}^{(1)}\left(\bar{D}_{1}\right):\left\|v_{h}\right\|_{L^{2}\left(D_{1}\right)} \leq q\right\}$, if $\int_{D_{1}} F_{h}\left(\chi_{h}\right) \chi_{h} d x>0$ for $\left\|\chi_{h}\right\|_{L^{2}\left(D_{1}\right)}=q$. On account of the assumptions $v_{s h} \in S_{Q}^{*}$ and $\chi_{h} \in S_{P}$, it follows that there exists a constant $C_{1}=C_{1}(P, Q, K)$ such that $\int_{D_{1}} a_{1} J_{h}^{n}\left(\chi_{h}\right) \chi_{h} d x \leq$ $C_{1}\left\|\chi_{h}\right\|_{L^{2}\left(D_{1}\right)}$. Hence,

$$
\begin{aligned}
\int_{D_{1}} F_{h}\left(\chi_{h}\right) \chi_{h} d x \geq & \left\|\chi_{h}\right\|_{L^{2}\left(D_{1}\right)}^{2}-\left\|u_{h}^{n-1}\right\|_{L^{2}\left(D_{1}\right)}^{2} \\
& +\Delta t k_{0}\left\|\frac{d \chi_{h}^{n}}{d x}\right\|_{L^{2}\left(D_{1}\right)}^{2}-\Delta t C_{1}\left\|\chi_{h}\right\|_{L^{2}\left(D_{1}\right)} \\
& \geq\left\|\chi_{h}\right\|_{L^{2}\left(D_{1}\right)}^{2}-\left\|u_{h}^{n-1}\right\|_{L^{2}\left(D_{1}\right)}^{2}-\Delta t C_{1}\left(1+\left\|\chi_{h}\right\|_{L^{2}\left(D_{1}\right)}\right)\left\|\chi_{h}\right\|_{L^{2}\left(D_{1}\right)} .
\end{aligned}
$$

Then, taking $\Delta t \leq \Delta t_{0}<1 / C_{1}, \int_{D_{1}} F_{h}\left(\chi_{h}\right) \chi_{h} d x$ is positive for $\left\|\chi_{h}\right\|_{L^{2}\left(D_{1}\right)}$ sufficiently large. This shows the existence of the solution $u_{h}^{n} \in V_{h}^{(1)}\left(\bar{D}_{1}\right)$. Next, we prove the uniqueness. To this end, we consider that there exist $X$ and $Y \in V_{h}^{(1)}\left(\bar{D}_{1}\right)$ satisfying (75), so

$$
\int_{D_{1}}(X-Y) w_{h} d x+\Delta t \int_{D_{1}} k_{1} \frac{d(X-Y)}{d x} \frac{d w_{h}}{d x} d x
$$




$$
=\Delta t \int_{D_{1}} a_{1}\left(J_{h}^{n}(X)-J_{h}^{n}(Y)\right) w_{h} d x \quad \forall w_{h} \in V_{h}^{(1)}\left(\bar{D}_{1}\right) .
$$

Setting $w_{h}=X-Y$ and invoking the arguments of Lemmas 5 and 6 yields

$$
\|X-Y\|_{L^{2}\left(D_{1}\right)}^{2}+k_{1} \Delta t\left\|\frac{d(X-Y)}{d x}\right\|_{L^{2}\left(D_{1}\right)}^{2} \leq \Delta t C_{2}\|X-Y\|_{L^{2}\left(D_{1}\right)}^{2},
$$

where the constant $C_{2}=C_{2}(P, Q, K)$. Thus, taking $\Delta t \leq \Delta t_{0}<1 / C_{2}$ it follows that $X=Y$. It remains to prove the existence and uniqueness of $v_{h \Delta r}^{n}$, but the arguments to be used for such a proof are the same as for $u_{h}^{n}$, so we omit them.

\subsection{Error estimates for the fully discrete solution}

As in Sect. 4.3, we write for $t=t_{n}$

$$
\left\{\begin{array}{l}
u^{n}-u_{h}^{n}=\rho_{u}^{n}+\theta_{u}^{n}, \\
v^{n}-v_{h \Delta r}^{n}=v^{n}-I_{0}^{x} v^{n}+I_{0}^{x} \rho_{v}^{n}+\theta_{v}^{n}
\end{array}\right.
$$

Theorem 17 Let $\left(u_{h}^{n}, v_{h \Delta r}^{n}, \phi_{1}^{n}, \phi_{2}^{n}\right)$ be the solution to (75)-(78). Then, under proper regularity assumptions there exists a constant $C$ such that for $\Delta t$ small

$$
\begin{aligned}
& \left\|u^{n}-u_{h}^{n}\right\|_{L^{2}\left(D_{1}\right)}^{2}+\left\|v^{n}-v_{h \Delta r}^{n}\right\|_{L^{2}\left(D_{2}, L_{r}^{2}\left(0, R_{s}(\cdot)\right)\right)}^{2} \\
& +\Delta t \sum_{j=1}^{t_{n}}\left\|\Phi^{j}-\Phi_{h}^{j}\right\|_{V}^{2} d \tau \leq C\left(h^{2}+\Delta r^{2}+\Delta t^{2}\right) .
\end{aligned}
$$

The constant $C$ is of the form $C(\Gamma) \exp \left(C\left(k_{1}, k_{2}\right) t_{n}, C(\Gamma)\right.$ being another constant that depends on the exact solution $\left(u, v, \phi_{1}, \phi_{2}\right)$, see (87) below.

Proof Since $\rho_{u}^{n}, v^{n}-I_{0}^{x} v^{n}+I_{0}^{x} \rho_{v}^{n}$ and $\left\|\Phi^{j}-\Phi_{h}^{j}\right\|_{V}^{2}$ are estimated as in Sect. 4.3, we shall address our attention to the estimations for $\theta_{u}^{n}$ and $\theta_{v}^{n}$. We start with the calculation for $\theta_{v}^{n}$. For this purpose, we recast (64) for $t=t_{n}$ as

$$
\begin{aligned}
& \int_{D_{2}} \int_{0}^{R_{S}(x)}\left(\widetilde{\partial}_{t} I_{0}^{x} v^{n} w+k_{2} \frac{\partial I_{0}^{x} v^{n}}{\partial r} \frac{\partial w}{\partial r}\right) r^{2} d r d x \\
& =-\int_{D_{2}} I_{0}^{x}\left(R_{s}^{2}(x) a_{2}^{-1}(x) F^{-1} J^{n} w\left(x ; R_{s}(x)\right)\right) d x \\
& \quad+\int_{D_{2}} \int_{0}^{R_{s}(x)}\left(\widetilde{\partial}_{t} I_{0}^{x} v^{n} w-\frac{\partial I_{0}^{x} v^{n}}{\partial t} w\right) r^{2} d r d x .
\end{aligned}
$$


Setting, as we did in Sect. 4.3, $v_{h \Delta r}^{n}=I_{0}^{x} v^{n}-\left(I_{0}^{x} \rho_{v}^{n}+\theta_{v}^{n}\right)$ in (76) and using ( 32) and (84) yields for $t=t_{n}$

$$
\begin{aligned}
& \int_{D_{2}} \int_{0}^{R_{s}(x)}\left(\tilde{\partial}_{t} \theta_{v}^{n} w_{h \Delta r}+k_{2} \frac{\partial \theta_{v}^{n}}{\partial r} \frac{\partial w_{h \Delta r}}{\partial r}\right) r^{2} d r d x \\
& =\lambda \int_{D_{2}} \int_{0}^{R_{s}(x)} I_{0}^{x} \rho_{v}^{n} w_{h \Delta r} r^{2} d r d x-\int_{D_{2}} \int_{0}^{R_{s}(x)} \tilde{\partial}_{t} I_{0}^{x} \rho_{v}^{n} w_{h \Delta r} r^{2} d r d x \\
& \quad-\int_{D_{2}} I_{h}^{0}\left(R_{s}^{2}(x) a_{2}^{-1}(x) F^{-1}\left(J^{n}(x)-J_{h}^{n}(x)\right) w_{h s}(x)\right) d x \\
& +\int_{D_{2}}\left(R_{s}^{2}(x) a_{2}^{-1}(x) F^{-1} J_{h}^{n}(x)-I_{h}^{0}\left(R_{s}^{2}(x) a_{2}^{-1}(x) F^{-1} J_{h}^{n}(x)\right)\right) w_{h s}(x) d x \\
& -\int_{D_{2}} \int_{0}^{R_{s}(x)}\left(\widetilde{\partial}_{t} I_{0}^{x} v^{n}-\frac{\partial I_{0}^{x} v^{n}}{\partial t}\right) r^{2} d r d x
\end{aligned}
$$

Letting $w_{h \Delta r}=\theta_{v}^{n}, w_{h s}=\theta_{v s}^{n}$, and noting that for $a$ and $b$ real numbers, $2(a-b) b=$ $a^{2}-b^{2}-(a-b)^{2}$, it follows that

$$
\begin{aligned}
\frac{1}{2} \widetilde{\partial}_{t} & \left\|\theta_{v}^{n}\right\|_{L^{2}\left(D_{2}, L_{r}^{2}\left(0, R_{s}(\cdot)\right)\right)}^{2}+\underline{k_{2}}\left\|\frac{\partial \theta_{v}^{n}}{\partial r}\right\|_{L^{2}\left(D_{2}, L_{r}^{2}\left(0, R_{s}(\cdot)\right)\right)}^{2} \\
\leq & \lambda\left\|I_{0}^{x} \rho_{v}^{n}\right\|_{L^{2}\left(D_{2}, L_{r}^{2}\left(0, R_{s}(\cdot)\right)\right)}\left\|\theta_{v}^{n}\right\|_{L^{2}\left(D_{2}, L_{r}^{2}\left(0, R_{s}(\cdot)\right)\right)} \\
& +\left\|\widetilde{\partial}_{t} I_{0}^{x} \rho_{v}^{n}\right\|_{L^{2}\left(D_{2}, L_{r}^{2}\left(0, R_{s}(\cdot)\right)\right)}\left\|\theta_{v}^{n}\right\|_{L^{2}\left(D_{2}, L_{r}^{2}\left(0, R_{s}(\cdot)\right)\right)} \\
& +C\left\|I_{h}^{0}\left(\left(J^{n}-J_{h}^{n}\right) \theta_{v s}^{n}\right)\right\|_{L^{1}\left(D_{2}\right)}+C\left\|\left(J_{h}^{n}-I_{h}^{0} J_{h}^{n}\right) \theta_{v s}^{n}\right\|_{L^{1}\left(D_{2}\right)} \\
& +\left\|\widetilde{\partial}_{t} I_{0}^{x} v^{n}-\frac{\partial I_{0}^{x} v^{n}}{\partial t}\right\|_{L^{2}\left(D_{2}, L_{r}^{2}\left(0, R_{s}(\cdot)\right)\right)}\left\|\theta_{v}^{n}\right\|_{L^{2}\left(D_{2}, L_{r}^{2}\left(0, R_{s}(\cdot)\right)\right)} \equiv \sum_{i=1}^{5} R_{i}^{n} .
\end{aligned}
$$

We bound the right hand side of this inequality applying the same arguments as in (66). Thus, we have that

$$
\begin{aligned}
& R_{1}^{n} \leq C \Delta r^{4}\left\|v^{n}\right\|_{L^{2}\left(D_{2}, H_{r}^{2}\left(0, R_{s}(\cdot)\right)\right)}^{2}+C\left\|\theta_{v}^{n}\right\|_{L^{2}\left(D_{2}, L_{r}^{2}\left(0, R_{s}(\cdot)\right)\right)}^{2} d t+C\left\|\theta_{v}^{n}\right\|_{L^{2}\left(D_{2}, L_{r}^{2}\left(0, R_{s}(\cdot)\right)\right)}^{2} . \\
& R_{2}^{n} \leq \frac{C \Delta r^{4}}{\Delta t} \int_{t_{n-1}}^{t_{n}}\left\|\frac{\partial v}{\partial t}\right\|_{L^{2}\left(D_{2}, H_{r}^{2}\left(0, R_{s}(\cdot)\right)\right)}^{2} d t\left\|\frac{\partial \theta_{v}^{n}}{\partial r}\right\|_{L^{2}\left(D_{2}, L_{r}^{2}\left(0, R_{s}(\cdot)\right)\right)}^{2} . \\
& R_{3}^{n} \leq C\left\|J^{n}-J_{h}^{n}\right\|_{L^{2}\left(D_{2}\right)}^{2}+C(\epsilon)\left\|\theta_{v}^{n}\right\|_{L^{2}\left(D_{2}, L_{r}^{2}\left(0, R_{s}(\cdot)\right)\right)}^{2}+\epsilon \|\left.\frac{\partial \theta_{v}^{n}}{\partial r}\right|_{L^{2}\left(D_{2}, L_{r}^{2}\left(0, R_{s}(\cdot)\right)\right)} ^{2} \\
& R_{4}^{n} \leq C\left\|J^{n}-J_{h}^{n}\right\|_{L^{2}\left(D_{2}\right)}^{2}+C(\epsilon)\left\|\theta_{v}^{n}\right\|_{L^{2}\left(D_{2}, L_{r}^{2}\left(0, R_{s}(\cdot)\right)\right)}^{2}+\epsilon \\
&+C h^{2}\left\|\frac{\partial J^{n}}{\partial x}\right\|_{L^{2}\left(D_{2}\right)}^{2} .
\end{aligned}
$$


In both $R_{3}^{n}$ and $R_{4}^{n}$ the term $C\left\|J^{n}-J_{h}^{n}\right\|_{L^{2}\left(D_{2}\right)}^{2}$ is bounded by Lemma 11 for $t=t_{n}$; thus, using the notation $\Gamma=\left(u, v, v_{s}, \phi_{1}, \phi_{2}\right)$, we can set that

$$
\begin{aligned}
C\left\|J^{n}-J_{h}^{n}\right\|_{L^{2}\left(D_{2}\right)}^{2} \leq & C(\Gamma)\left(h^{2}+\Delta r^{2}\right)+C\left\|\theta_{u}^{n}\right\|_{L^{2}\left(D_{1}\right)}^{2} \\
& +C(\epsilon)\left\|\theta_{v}^{n}\right\|_{L^{2}\left(D_{2}, L_{r}^{2}\left(0, R_{s}(\cdot)\right)\right)}^{2}+\epsilon\left\|\frac{\partial \theta_{v}^{n}}{\partial r}\right\|_{L^{2}\left(D_{2}, L_{r}^{2}\left(0, R_{s}(\cdot)\right)\right)}^{2}
\end{aligned}
$$

where the constant $C(\Gamma)$ is given as

$$
\begin{aligned}
C(\Gamma)= & C \max \left(\left\|\phi_{1}\right\|_{L^{\infty}\left(0, T_{\text {end }} ; H^{2}\left(D_{1}\right)\right)},\left\|\phi_{2}\right\|_{L^{\infty}\left(0, T_{\text {end }} ; H^{2}\left(D_{2}\right)\right)},\left\|v_{s}\right\|_{L^{\infty}\left(0, T_{\text {end }} ; H^{1}\left(D_{2}\right)\right)},\right. \\
& \left.\|u\|_{L^{\infty}\left(0, T_{\text {end }} ; H^{2}\left(D_{1}\right)\right)},\|v\|_{L^{\infty}\left(0, T_{\text {end }} ; L^{2}\left(D_{2} ; H_{r}^{2}\left(0, R_{s}(\cdot)\right)\right)\right)}\right) .
\end{aligned}
$$

Hence, we can write

$$
\begin{aligned}
R_{3}^{n}+R_{4}^{n} \leq & C(\Gamma)\left(h^{2}+\Delta r^{2}\right)+C h^{2}\left\|\frac{\partial J^{n}}{\partial x}\right\|_{L^{2}\left(D_{2}\right)}^{2}+C\left\|\theta_{u}^{n}\right\|_{L^{2}\left(D_{1}\right)}^{2} \\
& +C(\epsilon)\left\|\theta_{v}(t)\right\|_{L^{2}\left(D_{2}, L_{r}^{2}\left(0, R_{s}(\cdot)\right)\right)}^{2}+\epsilon\left\|\frac{\partial \theta_{v}(t)}{\partial r}\right\|_{L^{2}\left(D_{2}, L_{r}^{2}\left(0, R_{s}(\cdot)\right)\right)}^{2}
\end{aligned}
$$

To estimate the term $R_{5}^{n}$, we notice that by approximation theory

$$
\left\|\widetilde{\partial}_{t} I_{0}^{x} v^{n}-\frac{\partial I_{0}^{x} v^{n}}{\partial t}\right\|_{L^{2}\left(D_{2}, L_{r}^{2}\left(0, R_{s}(\cdot)\right)\right)} \leq C\left\|\widetilde{\partial}_{t} v^{n}-\frac{\partial v^{n}}{\partial t}\right\|_{L^{2}\left(D_{2}, L_{r}^{2}\left(0, R_{s}(\cdot)\right)\right)}
$$

and

$$
\widetilde{\partial}_{t} v^{n}-\frac{\partial v^{n}}{\partial t}=\frac{-1}{\Delta t} \int_{t_{n-1}}^{t_{n}}\left(t-t_{n-1}\right) \frac{\partial^{2} v}{\partial t^{2}} d t
$$

so,

$$
\left\|\widetilde{\partial}_{t} I_{0}^{x} v^{n}-\frac{\partial I_{0}^{x} v^{n}}{\partial t}\right\|_{L^{2}\left(D_{2}, L_{r}^{2}\left(0, R_{s}(\cdot)\right)\right)} \leq C\left(\Delta t \int_{t_{n-1}}^{t_{n}}\left\|\frac{\partial^{2} v}{\partial t^{2}}\right\|_{L^{2}\left(D_{2}, L_{r}^{2}\left(0, R_{s}(\cdot)\right)\right)}^{2} d t\right)^{1 / 2}
$$

Applying Young inequality yields

$$
R_{5}^{n} \leq C \Delta t \int_{t_{n-1}}^{t_{n}}\left\|\frac{\partial^{2} v}{\partial t^{2}}\right\|_{L^{2}\left(D_{2}, L_{r}^{2}\left(0, R_{s}(\cdot)\right)\right)}^{2} d t+C\left\|\theta_{v}^{n}\right\|_{L^{2}\left(D_{2}, L_{r}^{2}\left(0, R_{s}(\cdot)\right)\right)}^{2}
$$


Collecting these bounds in (85) and letting $\epsilon=\underline{k_{2}} / 2$ yields

$$
\begin{aligned}
& \left\|\theta_{v}^{n}\right\|_{L^{2}\left(D_{2}, L_{r}^{2}\left(0, R_{s}(\cdot)\right)\right)}^{2}+\Delta t \underline{k_{2}}\left\|\frac{\partial \theta_{v}^{n}}{\partial r}\right\|_{L^{2}\left(D_{2}, L_{r}^{2}\left(0, R_{s}(\cdot)\right)\right)}^{2} \leq\left\|\theta_{v}^{n-1}\right\|_{L^{2}\left(D_{2}, L_{r}^{2}\left(0, R_{s}(\cdot)\right)\right)}^{2}+F_{v}^{n} \\
& +C\left(\underline{\left.k_{2}\right)} \Delta t\left(\left\|\theta_{v}^{n}\right\|_{L^{2}\left(D_{2}, L_{r}^{2}\left(0, R_{s}(\cdot)\right)\right)}^{2}+\left\|\theta_{u}^{n}\right\|_{L^{2}\left(D_{1}\right)}^{2}\right)\right.
\end{aligned}
$$

where

$$
\begin{aligned}
F_{v}^{n}= & \Delta t\left(C(\Gamma)\left(h^{2}+\Delta r^{2}\right)+C h^{2}\left\|\frac{\partial J^{n}}{\partial x}\right\|_{L^{2}\left(D_{2}\right)}^{2}+C \Delta r^{4}\left\|v^{n}\right\|_{L^{2}\left(D_{2}, H_{r}^{2}\left(0, R_{s}(\cdot)\right)\right)}^{2}\right) \\
& +C \Delta r^{4} \int_{t_{n-1}}^{t_{n}}\left\|\frac{\partial v}{\partial t}\right\|_{L^{2}\left(D_{2}, H_{r}^{2}\left(0, R_{s}(\cdot)\right)\right)}^{2} d t+C \Delta t^{2} \int_{t_{n-1}}^{t_{n}}\left\|\frac{\partial^{2} v}{\partial t^{2}}\right\|_{L^{2}\left(D_{2}, L_{r}^{2}\left(0, R_{s}(\cdot)\right)\right)}^{2} d t .
\end{aligned}
$$

To calculate an estimate for $\theta_{u}^{n}$, we observe that subtracting (75) from (10) and setting $\theta_{u}^{n}=e_{u}^{n}-\rho_{u}^{n}$ it follows that

$$
\begin{gathered}
\int_{D_{1}} \widetilde{\partial}_{t} \theta_{u}^{n} w_{h} d x+\int_{D_{1}} k_{1} \frac{d \theta_{u}^{n}}{d x} \frac{d w_{h}}{d x} d x=\lambda \int_{D_{1}} \rho_{u}^{n} w_{h} d x-\int_{D_{1}} \partial \rho_{u}^{n} w_{h} d x \\
+\int_{D_{1}} a_{1}\left(J^{n}-J_{h}^{n}\right) w_{h} d x+\int_{D_{1}}\left(\widetilde{\partial}_{t} u^{n}-\frac{\partial u^{n}}{\partial t}\right) w_{h} d x .
\end{gathered}
$$

Letting $w_{h}=\theta_{u}^{n}$ yields

$$
\begin{aligned}
& \frac{1}{2} \widetilde{\partial}_{t}\left\|\theta_{u}^{n}\right\|_{L^{2}\left(D_{1}\right)}^{2}+\underline{k_{1}}\left\|\frac{d \theta_{u}^{n}}{d x}\right\|_{L^{2}\left(D_{1}\right)}^{2} \leq C\left(\left\|\rho_{u}^{n}\right\|_{L^{2}\left(D_{1}\right)}^{2}+\frac{1}{\Delta t} \int_{t_{n-1}}^{t_{n}}\left\|\frac{\partial \rho_{u}}{\partial t}\right\|_{L^{2}\left(D_{1}\right)}^{2} d t\right) \\
& \quad+C \Delta t \int_{t_{n-1}}^{t_{n}}\left\|\frac{\partial^{2} u}{\partial^{2} t}\right\|_{L^{2}\left(D_{1}\right)}^{2} d t+C\left\|J^{n}-J_{h}^{n}\right\|_{L^{2}\left(D_{1}\right)}^{2}+C\left\|\theta_{u}^{n}\right\|_{L^{2}\left(D_{1}\right)}^{2} \cdot
\end{aligned}
$$

Then by virtue of (29) and Lemma 11 it follows that

$$
\begin{aligned}
& \left\|\theta_{u}^{n}(t)\right\|_{L^{2}\left(D_{1}\right)}^{2}+\Delta t \underline{k_{1}}\left\|\frac{d \theta_{u}^{n}}{d x}\right\|_{L^{2}\left(D_{1}\right)}^{2} \leq\left\|\theta_{u}^{n-1}(t)\right\|_{L^{2}\left(D_{1}\right)}^{2}+F_{u}^{n} \\
& +C\left(\underline{k_{1}}, \underline{\left.k_{2}\right)} \Delta t\left(\left\|\theta_{v}^{n}\right\|_{L^{2}\left(D_{2}, L_{r}^{2}\left(0, R_{s}(\cdot)\right)\right)}^{2}+\left\|\theta_{u}^{n}\right\|_{L^{2}\left(D_{1}\right)}^{2}\right)+\frac{k_{2}}{2}\left\|\frac{\partial \theta_{v}^{n}}{\partial r}\right\|_{L^{2}\left(D_{2}, L_{r}^{2}\left(0, R_{s}(\cdot)\right)\right)}^{2}\right.
\end{aligned}
$$


where

$$
F_{u}^{n}=\Delta t C(\Gamma)\left(h^{2}+\Delta r^{2}\right)+C h^{4} \int_{t_{n-1}}^{t_{n}}\left\|\frac{\partial u}{\partial t}\right\|_{H^{2}\left(D_{1}\right)}^{2} d t+C \Delta t^{2} \int_{t_{n-1}}^{t_{n}}\left\|\frac{\partial^{2} u}{\partial^{2} t}\right\|_{L^{2}\left(D_{1}\right)}^{2} d t
$$

It remains to estimate $\left\|\Phi^{n}-\Phi_{h}^{n}\right\|_{V}$. Returning to the proof of Theorem 14 we have that for $t=t_{n}$

$$
\begin{aligned}
& \left\|\Phi^{n}-\Phi_{h}^{n}\right\|_{V}^{2} \leq C(\Gamma) h^{2}+C(\epsilon)\left(\left\|\theta_{u}^{n}\right\|_{L^{2}\left(D_{1}\right)}^{2}+\left\|\theta_{v}^{n}\right\|_{L^{2}\left(D_{2}, L_{r}^{2}\left(0, R_{s}(\cdot)\right)\right)}^{2}\right) \\
& +\epsilon\left\|\frac{\partial \theta_{v}^{n}}{\partial r}\right\|_{L^{2}\left(D_{2}, L_{r}^{2}\left(0, R_{S}(\cdot)\right)\right)}^{2} \cdot
\end{aligned}
$$

Thus, setting $\epsilon=\underline{k_{2}} / 2$ in (92) and adding (88), (90) and (92) we obtain that

$$
\begin{aligned}
& \left(1-C\left(\underline{k_{1}}, \underline{k_{2}}\right) \Delta t\right)\left(\left\|\theta_{u}^{n}\right\|_{L^{2}\left(D_{1}\right)}^{2}+\left\|\theta_{v}^{n}\right\|_{L^{2}\left(D_{2}, L_{r}^{2}\left(0, R_{s}(\cdot)\right)\right)}^{2}\right) \\
& +\Delta t\left\|\Phi^{n}-\Phi_{h}^{n}\right\|_{V}^{2} \leq F_{v}^{n}+F_{u}^{n} \\
& +\left\|\theta_{u}^{n-1}\right\|_{L^{2}\left(D_{1}\right)}^{2}+\left\|\theta_{v}^{n-1}\right\|_{L^{2}\left(D_{2}, L_{r}^{2}\left(0, R_{S}(\cdot)\right)\right)}^{2}
\end{aligned}
$$

For $\Delta t$ small

$$
\begin{gathered}
\left\|\theta_{u}^{n}\right\|_{L^{2}\left(D_{1}\right)}^{2}+\left\|\theta_{v}^{n}\right\|_{L^{2}\left(D_{2}, L_{r}^{2}\left(0, R_{S}(\cdot)\right)\right)}^{2}+\Delta t\left\|\Phi^{n}-\Phi_{h}^{n}\right\|_{V}^{2} \leq C\left(F_{v}^{n}+F_{u}^{n}\right) \\
\left.\left(1+C \underline{\left(k_{1}\right.}, \underline{k_{2}}\right) \Delta t\right)\left(\left\|\theta_{u}^{n-1}\right\|_{L^{2}\left(D_{1}\right)}^{2}+\left\|\theta_{v}^{n-1}\right\|_{L^{2}\left(D_{2}, L_{r}^{2}\left(0, R_{S}(\cdot)\right)\right)}^{2}\right) .
\end{gathered}
$$

Hence, by repeated application and taking $\left\|\theta_{u}^{0}\right\|_{L^{2}\left(D_{1}\right)}^{2}+\left\|\theta_{v}^{0}\right\|_{L^{2}\left(D_{2}, L_{r}^{2}\left(0, R_{s}(\cdot)\right)\right)}^{2}=0$, it results that

$$
\begin{aligned}
& \left\|\theta_{u}^{n}\right\|_{L^{2}\left(D_{1}\right)}^{2}+\left\|\theta_{v}^{n}\right\|_{L^{2}\left(D_{2}, L_{r}^{2}\left(0, R_{s}(\cdot)\right)\right)}^{2}+\Delta t \sum_{j=1}^{n}\left\|\Phi^{j}-\Phi_{h}^{j}\right\|_{V}^{2} \\
& \leq C \sum_{j=1}^{n} \frac{F_{v}^{j}+F_{u}^{j}}{\left.\left(1+C\left(\underline{k_{1}}, \underline{k_{2}}\right)\right) \Delta t\right)^{j-n}} .
\end{aligned}
$$

Noting that $\left(1+C\left(k_{1}, k_{2}\right) \Delta t\right) \leq e^{C\left(k_{1}, k_{2}\right) \Delta t}$, then we can write

$$
\begin{aligned}
& \sum_{j=1}^{n} \frac{F_{v}^{j}+F_{u}^{j}}{\left(1+C\left(\underline{k_{1}}, \underline{k_{2}}\right) \Delta t\right)^{j-n}} \leq e^{C\left(\underline{k_{1}}, \underline{k_{2}}\right) t_{n}} \sum_{j=1}^{n} F_{v}^{j}+F_{u}^{j} \quad \text { (by (89) and (91)) } \\
& \quad \leq C(\Gamma) e^{C\left(\underline{k_{1}}, \underline{\left.k_{2}\right)} t_{n}\right.}\left(h^{2}+\Delta r^{2}+\Delta t^{2}\right) .
\end{aligned}
$$

This completes the proof 
Acknowledgements This research has been partially funded by grant PGC-2018-097565-B100 of Ministerio de Ciencia, Innovación y Universidades of Spain.

Funding Open Access funding provided thanks to the CRUE-CSIC agreement with Springer Nature.

Open Access This article is licensed under a Creative Commons Attribution 4.0 International License, which permits use, sharing, adaptation, distribution and reproduction in any medium or format, as long as you give appropriate credit to the original author(s) and the source, provide a link to the Creative Commons licence, and indicate if changes were made. The images or other third party material in this article are included in the article's Creative Commons licence, unless indicated otherwise in a credit line to the material. If material is not included in the article's Creative Commons licence and your intended use is not permitted by statutory regulation or exceeds the permitted use, you will need to obtain permission directly from the copyright holder. To view a copy of this licence, visit http://creativecommons.org/licenses/by/4.0/.

\section{References}

1. Bermejo, R., Galán del Sastre, P.: An implicit-explicit Runge-Kutta-Chebyshev finite element method for the nonlinear Lithium-ion battery equations. Appl. Math. Comput. 36, 398-420 (2019)

2. Ciarlet, P.G.: The Finite Element Method for Elliptic Prolems. North Holland, Amsterdand (1978)

3. Doyle, M., Fuller, T.F., Newman, J.: Modeling of galvanostatic charge and discharge of the lithium/polymer/insertion cell. J. Electrochem. Soc. 140, 1526-1533 (1993)

4. Díaz, J.I., Gómez-Castro, D., Ramos, A.M.: On the well-posedness of a multiscale mathematical model for Lithium-ion batteries. Adv. Nonlinear Anal. 8, 1132-1157 (2019)

5. Eriksson, K., Thomée, V.: Galerkin methods for singular value problems in one space dimension. Math. Comp. 42, 345-367 (1984)

6. Farkas, Z., Faragó, I., Kriston, Á., Pfrang, A.: Improvement of the accuracy of multi-scale models of Li-ion batteries by applying operating splitting techniques. J. Comput. Appl. Math. 310, 59-79 (2017)

7. Gilbert, D., Trudinger, N.: Elliptic Partial Differential Equations of Second Order. Springer, Heidelberg (1977)

8. Girault, V., Raviart, P.-A.: Finite Element Methods for Navier-Stokes Equations. Springer, Heidelberg (1986)

9. Kosch, S., Sturm, J., Schuster, J., Mulder, G., Ayerbe, E., Jossem, A.: A computationally efficient multi-scale model for lithium-ion cells. J. Electrochem. Soc. 165, A2374-A2388 (2018)

10. Kröner, C.: A mathematical exploration of a PDE system for lithium-ion batteries, Ph.D. Thesis in Mathematics University of California, Berkeley (2016) 63 pp

11. Mazunder, S., Lu, J.: Faster-than-real-time simulation of Lithium-ion batteries with full spatial and temperal resolution. Int. J. Electrochem, 268747, 10 pp. ID 268747 (2013)

12. Newman, J.: Dualfoil 5.1 Newman research group webpage. http://www.cchem.berkeley.edu/jsngrp/

13. Newman, J., Thomas-Alyea, K.E.: Electrochemical Systems, 3rd edn. Wiley, Hoboken (2004)

14. Northrop, P.W.C., Pathak, M., Rife, D., De, S., Santhanagopalan, S., Subramanian, V.R.: Efficient simulation and model reformulation of two-dimensional electrochemical thermal behavior of Lithiumion batteries. J. Electrochem. Soc. 162, A940-A951 (2015)

15. Plett, G.L.: Battery Management System, vol. 1. Artech House, Boston (2015)

16. Schreiber, R., Eisenstat, S.: Finite element methods for spherically symmetric elliptic equations. SIAM J. Numer. Anal. 18, 546-558 (1981)

17. Smith, K.A., Wang, C.-Y.: Solid-state diffusion limitations on pulse operation of a Lithium-ion cell for hybrid electric vehicles. J. Power Sour. 161, 628-639 (2006)

18. Zeidler, E.: Nonlinear Functional Analysis and Its Applications IIB. Springer, New York (2013)

19. Wu, J., Xu, J., Zou, H.: On the well-posedness of a mathematical model for lithium-ion battery systems. Meth. Appl. Anal. 13, 275-298 (2006)

Publisher's Note Springer Nature remains neutral with regard to jurisdictional claims in published maps and institutional affiliations. 